Research Article

\title{
Ethnobotanical Study of Medicinal Plants Used by Traditional Healers to Treat Cancer-Like Symptoms in Eleven Districts, Ethiopia
}

\author{
Solomon Tesfaye (D), Anteneh Belete, Ephrem Engidawork, \\ Teferi Gedif $\mathbb{D}$, and Kaleab Asres \\ School of Pharmacy, College of Health Sciences, Addis Ababa University, Addis Ababa, Ethiopia \\ Correspondence should be addressed to Solomon Tesfaye; soltesfa2010@gmail.com
}

Received 10 December 2019; Revised 5 March 2020; Accepted 18 March 2020; Published 21 April 2020

Academic Editor: Sebastian Granica

Copyright (C) 2020 Solomon Tesfaye et al. This is an open access article distributed under the Creative Commons Attribution License, which permits unrestricted use, distribution, and reproduction in any medium, provided the original work is properly cited.

\begin{abstract}
There is no ethnobotanical study conducted specifically on medicinal plants traditionally used to treat cancer in Ethiopia. Yet, traditional herbalists in different parts of the country claim that they have been treating cancer-like symptoms using herbal remedies. The objective of this study was to document medicinal plants traditionally used to treat cancer-like symptoms in eleven districts, Ethiopia. Traditional herbalists were interviewed using semistructured questionnaires, and field visits were also carried out to collect claimed plants for identification purpose. Seventy-four traditional herbalists, who claimed that they knew about and/ or had used medicinal plants to treat cancer-like symptoms, were selected using the snowball method and interviewed. Herbalists used their intuition and relied on the chronicity, growth of external mass, and spreading of the disease to other parts of the body, as a means to characterize cancer symptoms. Furthermore, in some of the study districts, herbalists reported that they treat patients who had already been diagnosed in modern healthcare institutions prior to seeking help from them. The inventory of medicinal plants is summarized in a synoptic table, which contains the scientific and vernacular names of the plants, their geographical location, the parts of the plants, and the methods used to prepare the remedies. A total of 53 traditionally used anticancer plants, belonging to 30 families, were identified during the survey. The most frequently reported anticancer plants were Acmella caulirhiza Del (Asteraceae), Clematis simensis Fresen. (Ranunculaceae), Croton macrostachyus Del. (Euphorbiaceae), and Dorstenia barnimiana Schweinf. (Moraceae). Organizing traditional healers, documenting their indigenous knowledge, and scientifically validating it for the development of better cancer therapeutic agents constitute an urgent and important task for policymakers and scientists.
\end{abstract}

\section{Introduction}

Cancer is a complex disease that is very heterogenic and variable at cellular level and also differs from one patient to the other in its behaviour, development, and outcome [1]. Physical, metabolic, and behavioural variations of cancer cells from normal ones arise through the accumulation of genetic modifications and help them to proliferate rapidly, escape from host immune surveillance, and ultimately invade distant tissues [2]. Histopathological, genetic, and epigenetic and clinical outcome variations between and within different types of cancers have been the greatest challenge to understand the disease and develop novel therapies [3].

Surgery and radiation therapy were the most preferred means of treatment to control cancer before 1950 and after 1960, respectively [4]. Chemotherapy can be done before surgery to shrink the tumor or after surgery to kill the remaining cancer cells [5]. However, most of the chemotherapeutic drugs lack specificity and tend to rapidly damage normal dividing tissues, causing side effects such as immunosuppression, neurotoxicity, and hair loss [6]. Moreover, resistance has also reduced therapeutic efficacy of some anticancer chemotherapeutic drugs [7]. 
In order to address these limitations, tapping nature as a major source of chemically diverse novel anticancer compounds is a consistently proven track [8]. Screening natural products yield more hit with more "drug-like" characteristics (absorption and metabolism) as compared to screening of rationally designed compounds [9]. Furthermore, screening medicinal plants based on traditional use provides a higher chance of finding active plants relative to the random approach [10].

Ethiopia has a rich and diverse heritage of traditional medical practices, known for using plants to prepare more than $90 \%$ of the remedies [11]. In addition, the country has more than 6,500 higher plant species of which, around $12 \%$ are endemic [12]. Reports indicate that up to $80 \%$ of the population relies on traditional remedies as a primary source of health care [13]. Only few ethnobotanical reports from different agroecological zones of Ethiopia are available in the literature regarding medicinal plants used for cancer treatment. These include Bersama abyssinica, Buddleja polystachya, Clerodendrum myricoides, Dovyalis abyssinica, Ekebergia capensis, Myrsine melanophloeos, Olea capensis, Pentas lanceolata, Sideroxylon oxyacanthum, and Zingiber officinale [14]; Bidens macroptera, Clematis simensis, Ferula communis, and Punica granatum [15]; Rumex abyssinicus [16]; Zanthoxylum chalybeum [17]; Phytolacca dodecandra and Vinca rosea [18]; Kalanchoe lanceolata, Stephania abyssinica, and Vernonia hymenolepis [19]; Plumbago zeylanica [20-22]; Acalypha acrogyna, Carissa spinarum, Maytenus ovatus, and Salvia nilotica [23]; Croton macrostachyus [24]; and Dorstenia barnimiana [25, 26].

In view of this fact and considering the weak traditional recording and knowledge transfer system and an alarming rate of environmental degradation, finding anticancer plants and documenting their ethnobotanical information constitute an urgent and indispensable task. Therefore, the main aim of this study was to establish an inventory of medicinal plants traditionally used to treat cancer in eleven districts of Ethiopia.

\section{Materials and Methods}

2.1. Description of the Study Areas. This ethnobotanical study was conducted in four national regional states of Ethiopia: Oromia, Amhara, Afar, and Southern Nations, Nationalities, and People. The survey included different districts from each region, namely, Bale Robe and Goba from Oromia, Bahir Dar Zuria and Filiklik from Amhara, Gewane from Afar, and Wondo Genet, Sodo Zuria, Doyo Gena, North Bench, Mizan Aman, and Shako from Southern Nations, Nationalities, and People Regional State (Figure 1). These geographically, culturally, and agroecologically different study areas (Table 1) were selected mainly based on the availability of traditional healers and recommendations from health workers.

2.2. Data Collection. A team comprising a botanist and researchers from Addis Ababa University was set up, and health authorities were contacted for permission and identification of traditional herbalists living in each study area. Altogether, 117 traditional healers were approached using the snowball technique and 74 traditional healers who used herbs to manage cancer-like symptoms were selected. Ethnobotanical data were collected between January and August 2016, mainly through individual interviews with the selected traditional herbalists using a semistructured interview questionnaire. The questionnaire was prepared in Amharic language and translated to different local languages for traditional healers who do not speak Amharic. This questionnaire was designed to obtain information in the following areas: (i) general data on the informant, (ii) school attendance, (iii) use of plants for cancer treatment, (iv) source of the plant material, (v) part of the plant used, (vi) method of medicinal preparation, (vii) route of administration, and (viii) side effects.

A traditional healer for the purpose of this study is "a person who is recognized by the community in which s/he lives as competent to provide healthcare by using plants and plant products." Each traditional healer was approached, briefed about the purpose of the research, and asked for his/ her verbal consent in talking about cancer and its treatment. They were assured of the confidentiality of the information they provided. If plants were mentioned for their anticancer purposes, a botanical sample was collected. These specimens were pressed and preserved for later identification at the National Herbarium, Addis Ababa University, Addis Ababa, and a voucher specimen of each plant was deposited in the institute. All botanical names have been transcribed according to the nomenclature system used by the Plant List (http://www.theplantlist.org).

2.3. Data Analysis. The relative importance of medicinal plants used in the management of cancer-like symptoms in study areas was assessed using the relative frequency of citation (RFC), use value (UV), informants consensus factor (ICF), and cultural importance index (CI).

2.3.1. Relative Frequency of Citation (RFC). The RFC was calculated by dividing the number of informants that cite a particular plant species (FC) by the total number of informants in the survey $(N)$ [29]:

$$
\mathrm{RFC}=\frac{\mathrm{FC}}{N} .
$$

2.3.2. Use Value (UV). The use value demonstrates the relative importance of plant species to treat particular ailment, and it is determined by the following formula [30]:

$$
\mathrm{UV}=\sum \frac{U_{i}}{N_{i}}
$$

where "UV" stands for the use value of a species, " $U_{i}$ " stands for the number of use reports cited by informants for that plant species, and " $N_{i}$ " is the total number of informers who reported the particular plant species $i$. 


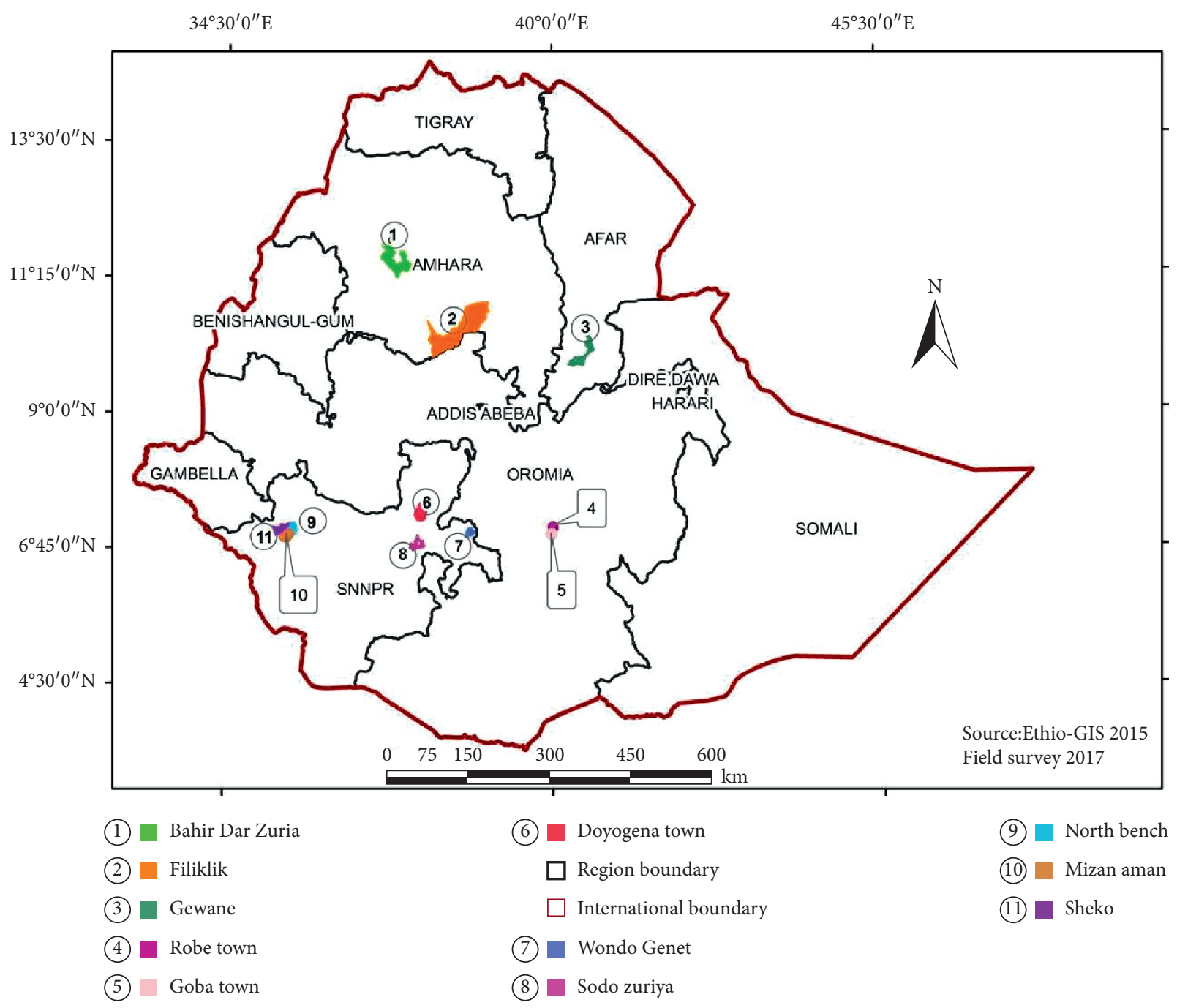

FIGURE 1: Map of Ethiopia showing the location of study districts.

2.3.3. Informant Consensus Factor (ICF). Informant consensus factor (ICF) was calculated to determine the homogeneity of the information collected about particular plant species to treat specific ailment. It was estimated using the following formula [31]:

$$
\mathrm{ICF}=\frac{\mathrm{Nur}-\mathrm{Nt}}{\mathrm{Nur}-1}
$$

where Nur is the number of use reports of informants for particular ailment category and $\mathrm{Nt}$ refers to the number of species used for the ailment category by all informants.

2.3.4. Cultural Importance Index (CI). Cultural importance index (CI) is calculated by the sum of the use reports (UR) of informants mentioning each species use (from $i_{1}$ to $i_{N}$ ) in each use category and adding all the UR of each category (from $u_{1}$ to $u_{\mathrm{NC}}$ ) divided by the total number of informants $N$. This index is determined by the following formula [29]:

$$
\mathrm{CI}_{i} \sum_{u=u_{1}}^{u_{\mathrm{NC}}} \sum_{i=i_{1}}^{i_{N}} \frac{\mathrm{UR} u_{i}}{N}
$$

where CI is an ethnobotanical index that indicates the spread of the use along with the diversity of uses of each species.

\section{Results}

The informants consisted of 66 male and 8 female traditional healers and they were divided into three age groups: 20-40, $41-60$, and $\geq 61$ years. Out of 74 interviewed traditional healers, most of them (N\%) were adults aged between 41 and 60 years. Majority of the respondents $(70.2 \%)$ gained their knowledge from family members and $82 \%$ of all interviewed respondents practiced ethnomedicine for more than 25 years. More than $70 \%$ of the respondents were either only at their primary level of education or did not have a formal education at all (Figure 2). Traditional healers usually used their intuition and relied on the chronicity and growth of external mass, as a means to diagnose cancer. Lumpy growth was the most commonly cited criteria used to diagnose cancer, followed by ulcerative wounds and bleeding ( Table 2). However, there were instances where some of the healers claimed to have treated patients already diagnosed with cancer at modern health institutions. Traditional 
TABle 1: Vegetation type, climatic condition, and demographic data of the study areas $[27,28]$ (source: National Meteorological Service Agency of Ethiopia).

\begin{tabular}{|c|c|c|c|c|c|c|c|c|c|}
\hline \multirow[b]{2}{*}{ District } & \multirow{2}{*}{$\begin{array}{l}\text { Distance } \\
\text { from } \\
\text { capital } \\
\text { city }(\mathrm{km})\end{array}$} & \multirow[b]{2}{*}{$\begin{array}{l}\text { Approximate } \\
\text { population } \\
(2015)\end{array}$} & \multirow[b]{2}{*}{$\begin{array}{c}\text { Number of } \\
\text { interviewed } \\
\text { healers }\end{array}$} & \multirow[b]{2}{*}{$\begin{array}{l}\text { Area size } \\
\left(\mathrm{km}^{2}\right)\end{array}$} & \multirow[b]{2}{*}{$\begin{array}{c}\text { Geographical } \\
\text { location }\end{array}$} & \multirow{2}{*}{$\begin{array}{c}\text { Average } \\
\text { elevation } \\
\text { above sea } \\
\text { level } \\
\text { (m.a.s.l) }\end{array}$} & \multirow[b]{2}{*}{$\begin{array}{l}\text { Vegetation } \\
\text { type }\end{array}$} & \multicolumn{2}{|c|}{$\begin{array}{c}\text { Climatic condition } \\
\text { (2014) }\end{array}$} \\
\hline & & & & & & & & $\begin{array}{l}\text { Annual } \\
\text { average } \\
\text { rainfall } \\
(\mathrm{mm})\end{array}$ & $\begin{array}{c}\text { Annual } \\
\text { average } \\
\text { temperature } \\
\text { range }\left({ }^{\circ} \mathrm{C}\right)\end{array}$ \\
\hline $\begin{array}{l}\text { Bale } \\
\text { Robe }\end{array}$ & 432 & 65,284 & 2 & 8.87 & $\begin{array}{l}7^{\circ} 07^{\prime} 11.65^{\prime \prime} \mathrm{N} \\
40^{\circ} 00^{\prime} 24.82^{\prime \prime} \mathrm{E}\end{array}$ & 2480 & DAF & 745.6 & $9.2-23.2$ \\
\hline Goba & 444 & 47,135 & 7 & 20.15 & $\begin{array}{l}7^{\circ} 00^{\prime} 41.66^{\prime \prime} \mathrm{N} \\
39^{\circ} 58^{\prime} 33.96^{\prime \prime} \mathrm{E}\end{array}$ & 2614 & DAF & 736.3 & $9.5-23.8$ \\
\hline $\begin{array}{l}\text { Bahir } \\
\text { Dar } \\
\text { Zuria }\end{array}$ & 578 & 206,708 & 16 & 1443.37 & $\begin{array}{l}11^{\circ} 34^{\prime} 27.15^{\prime \prime} \mathrm{N} \\
37^{\circ} 21^{\prime} 40.87^{\prime \prime} \mathrm{E}\end{array}$ & 1800 & $\begin{array}{c}\text { CTW, DAF, } \\
\text { and FLV/ } \\
\text { MFS }\end{array}$ & 1547.1 & $12.7-27.6$ \\
\hline Filiklik & 188 & 142,722 & 7 & 806.98 & $\begin{array}{c}10^{\circ} 02^{\prime} 12.74^{\prime \prime} \mathrm{N} \\
38^{\circ} 14^{\prime} 27.65^{\prime \prime} \mathrm{E} \\
10^{\circ} 29^{\prime} 59.99^{\prime \prime}\end{array}$ & 1853 & $\begin{array}{c}\text { CTW and } \\
\text { DAF }\end{array}$ & 880.2 & $12.9-22.0$ \\
\hline Gewane & 344 & 39,186 & 6 & 967.85 & $\begin{array}{c}\mathrm{N} \\
40^{\circ} 44^{\prime} 59.99^{\prime \prime} \mathrm{E}\end{array}$ & 568 & $\mathrm{ACB}$ & 586.7 & $19.5-36.7$ \\
\hline $\begin{array}{l}\text { Wondo } \\
\text { Genet }\end{array}$ & 270 & 196,277 & 12 & 226.45 & $\begin{array}{l}7^{\circ} 05^{\prime} 3.01^{\prime \prime} \mathrm{N} \\
38^{\circ} 37^{\prime} 8.02^{\prime \prime} \mathrm{E}\end{array}$ & 1742 & DAF & 928.7 & $15-29.6$ \\
\hline $\begin{array}{l}\text { Sodo } \\
\text { Zuria }\end{array}$ & 383 & 145,092 & 2 & 25.62 & $\begin{array}{l}6^{\circ} 51^{\prime} 10.11^{\prime \prime} \mathrm{N} \\
37^{\circ} 45^{\prime} 39.49^{\prime \prime} \mathrm{E}\end{array}$ & 1854 & $\begin{array}{l}\text { CTW and } \\
\text { DAF }\end{array}$ & 1569.2 & $14.8-25.2$ \\
\hline $\begin{array}{l}\text { Doyo } \\
\text { Gena }\end{array}$ & 258 & 95,393 & 14 & 130.57 & $\begin{array}{l}7^{\circ} 21^{\prime} 20.22^{\prime \prime} \mathrm{N} \\
37^{\circ} 47^{\prime} 07.15^{\prime \prime} \mathrm{E}\end{array}$ & 2300 & DAF & 1334.5 & $11-22.8$ \\
\hline $\begin{array}{l}\text { North } \\
\text { Bench }\end{array}$ & 587 & 126,308 & 4 & 392.65 & $\begin{array}{l}6^{\circ} 37^{\prime} 53.43^{\prime \prime} \mathrm{N} \\
35^{\circ} 33^{\prime} 56.83^{\prime \prime} \mathrm{E}\end{array}$ & 2367 & CTW & 1671.8 & $16-33.3$ \\
\hline $\begin{array}{l}\text { Mizan } \\
\text { Aman }\end{array}$ & 565 & 64,996 & 3 & 24.45 & $\begin{array}{c}6^{\circ} 59^{\prime} 37.13^{\prime \prime} \mathrm{N} \\
35^{\circ} 34^{\prime} 55.92^{\prime \prime} \mathrm{E}\end{array}$ & 1441 & $\begin{array}{l}\text { CTW and } \\
\text { MAF }\end{array}$ & 1963.7 & $14.8-28.8$ \\
\hline Shako & 617 & 51,195 & 1 & $48,089.63$ ha & $\begin{array}{l}7^{\circ} 33^{\prime} 42.37^{\prime \prime} \mathrm{N} \\
35^{\circ} 39^{\prime} 11.83^{\prime \prime} \mathrm{E}\end{array}$ & 1800 & $\begin{array}{l}\text { CTW and } \\
\text { MAF }\end{array}$ & 1906.9 & $11.4-22.4$ \\
\hline
\end{tabular}

Note. Vegetation type: DAF: dry evergreen Afromontane forest and grassland complex; CTW: Combretum-Terminalia woodland and wooded grassland; FLV/MFS: freshwater marshes and swamps, floodplains, and lake shore vegetation; ACB: Acacia-Commiphora woodland and bushland proper; MAF: moist evergreen Afromontane forest. m.a.s.l: meter above sea level; mm: millimeter; ${ }^{\circ} \mathrm{C}$ : degree Celsius; $\mathrm{km}^{2}$ : kilometer square.

healers identified cancer as "Nekersa" in Bahir Dar Zuria and Filiklik, "Naqarsa" in Bale Robe and Goba, "Sissac" in Gewane, "Xoka or Toka" in Doyo Gena, "Balamo" in Wondo Genet, "Kums or niamt" in North Bench, and "Kanser" in Sheko and Sodo Zuria district. Out of the 6 specific cancer types (skin, breast, lung, cervical, throat, and intestinal) claimed to be treated by the respondents, skin cancer was a dominant one followed by breast cancer.

A total of 53 plant species belonging to 30 families were reported for their anticancer use (Table 3 ). The result of this study showed that shrubs (49.1\%), herbs (33.9\%), trees (13.2\%), and climbers $(3.8 \%)$ were the main sources of potential anticancer medicinal plants. This study also indicated that leaves $(56.7 \%)$ were the most commonly used plant parts followed by roots $(21.7 \%)$, bark $(6.7 \%)$, stem $(1.7 \%)$, seeds $(1.7 \%)$, whole plant $(1.7 \%)$, leaves and roots $(5 \%)$, leaves or stem $(1.7 \%)$, and leaves or seeds (1.7\%) (Figure 3). Most of the reported plants occurred naturally in wild (96.2\%); however, cultivation was also a source (3.8\%). Reported medicinal plants have been traditionally claimed to be used to treat different types of ailments including cancer. However, only few have been scientifically investigated for their antiproliferative or cytotoxic activity (Table 4). While comparing the amount and distribution of anticancer plants in the past ten years, regardless of the study areas, all respondents believed that the amount and distribution of these plants are reduced.

In the current study, the highest UVs were recorded for Aloe spp. (6), Albizia schimperiana (4), Sida schimperiana (4), Achyranthes aspera (4), Brucea antidysenterica (4), Cleome brachycarpa (3), Leonotis ocymifolia (3), and Prunus africana (3). The lowest UVs were obtained for Acokanthera schimperi, Acmella caulirhiza, Cineraria abysinica, and Gnidia involucrata (Table 3). A total of 228 use reports have been documented and categorized into seven categories (Table 5). Among these, other ailments (46.3\%) and skin cancer $(26.5 \%)$ had the highest use reports. Furthermore, ICF values were also calculated and ranged from 0 to 0.42 . The highest ICF values were recorded in other ailments (0.42) and breast cancer (0.32) followed by skin cancer (0.23) category (Table 5). The other ailments category comprises of diseases such as stomach ache, malaria, wart, swelling, wounds, evil eye, toothache, bleeding, gastrointestinal disorder, headache, bone fracture, cough, snake bite, herpes simplex, tonsillitis, hypertension, dandruff, fever, and hemorrhoid. The ICF value of the remaining four categories (lung cancer, colon cancer, cervical cancer, and throat cancer) was zero. Quantitative ethnobotanical indexes such as RFC and CI were calculated in this study to 


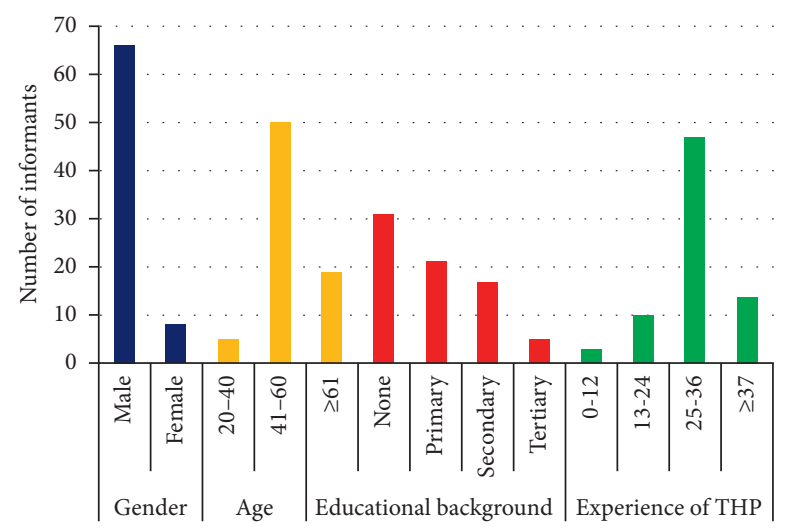

FIgURE 2: Demographic details of the interviewed informants.

TABLE 2: Symptoms that are used by traditional healers to diagnose cancer.

\begin{tabular}{|c|c|c|}
\hline Cancer types & Reported symptoms & Number of traditional healers \\
\hline \multirow{3}{*}{ Skin } & Lumpy growth & 32 \\
\hline & Spreading pea-sized growth & 1 \\
\hline & Ulcerative growth and oozing blood & 1 \\
\hline \multirow{5}{*}{ Breast } & Lumpy growth & 17 \\
\hline & Lumpy growth on one breast and progressive weight loss & 1 \\
\hline & Ulcerative wounds on breast & 5 \\
\hline & Ulcerative wounds on breast and swelling on armpit and neck & 1 \\
\hline & The patient was receiving anticancer treatment for breast cancer in hospital & 12 \\
\hline Cervical & Foul-smelling bloody vaginal discharge, pain during sexual intercourse, and weight loss & 1 \\
\hline Colon & Chronic rectal bleeding and weight loss & 1 \\
\hline Lung & Coughing up blood & 1 \\
\hline Throat & Coughing and swelling on the neck & 1 \\
\hline
\end{tabular}

analyze the ethnobotanical information. According to RFC values, Croton macrostachyus (0.1), Vernonia auriculifera (0.04), Clematis simensis (0.04), and Acmella caulirhiza (0.04) are the most frequently cited among all reported plants. Croton macrostachyus (0.16), Dorstenia barnimiana (0.12), and Aloe spp. (0.08) rank $1^{\text {st }}, 2^{\text {nd }}$, and $3^{\text {rd }}$ in position, respectively, according to the CI reference. Our result also shows that the Pearson correlation coefficient of RFC was positively and negatively correlated to CI and UV, respectively (Table 6).

Most of the reported remedies, prepared from these plants, were either applied topically $(50 \%)$ or taken orally (41.7\%). The remaining remedies were prepared to be administered either topically or orally $(3.3 \%)$, both topically and orally $(1.7 \%)$, and intranasally (1.7\%). Usually, fresh plants were finely chopped, dried, and pounded to powder form. Then, the powder of either one or the combination of more than one plant was either mixed with drinking water or pasted and applied topically. In other cases, fresh plant parts were decocted and taken orally or crushed and applied topically. Water was the main medium in preparation of most remedies and additives like honey, milk, and butter were also used. To determine the amount of plant parts used to prepare remedies, traditional healers used spoon, fingertip, and number (in case of fresh leaves). Adverse effects reported by respondents include vomiting, diarrhea, and skin ulcers.

\section{Discussion}

Despite the rich biodiversity of the study areas, broad acceptability, and centuries-old tradition of using traditional medicines, the number of anticancer plants reported in this study is far less than expected. As it was reported by different ethnobotanical studies conducted in different parts of Ethiopia, this could be attributed to the attitude of many traditional healers to guard their indigenous medical knowledge as a family secret and hence hesitant to share with the researchers $[13,32,73]$. Justifying the lower number of female traditional healers $(8,11 \%)$ participated in this study, these studies also inferred that traditional healers usually pass their knowledge to the first son of the family.

In this study, in agreement with the studies conducted in Fiche district [35], Ghimbi district [20], and Hawassa city [17] of Ethiopia, the predominant botanical families recorded, listing over 5 plant species each, were Asteraceae, Fabaceae, and Lamiaceae. This could be due to the fact that these families are the largest in the flora of Ethiopia and Eritrea [15, 21, 143]. Moreover, cytotoxicity studies conducted on different Mexican plants reported that the highest number of plant species with both in vitro and in vivo antineoplasic activities was from these families [20].

The highest UVs recorded in this study include Aloe spp. (6), Achyranthes aspera L. (4), Albizia schimperiana (4), Sida schimperiana (4), and Brucea antidysenterica (4). The 


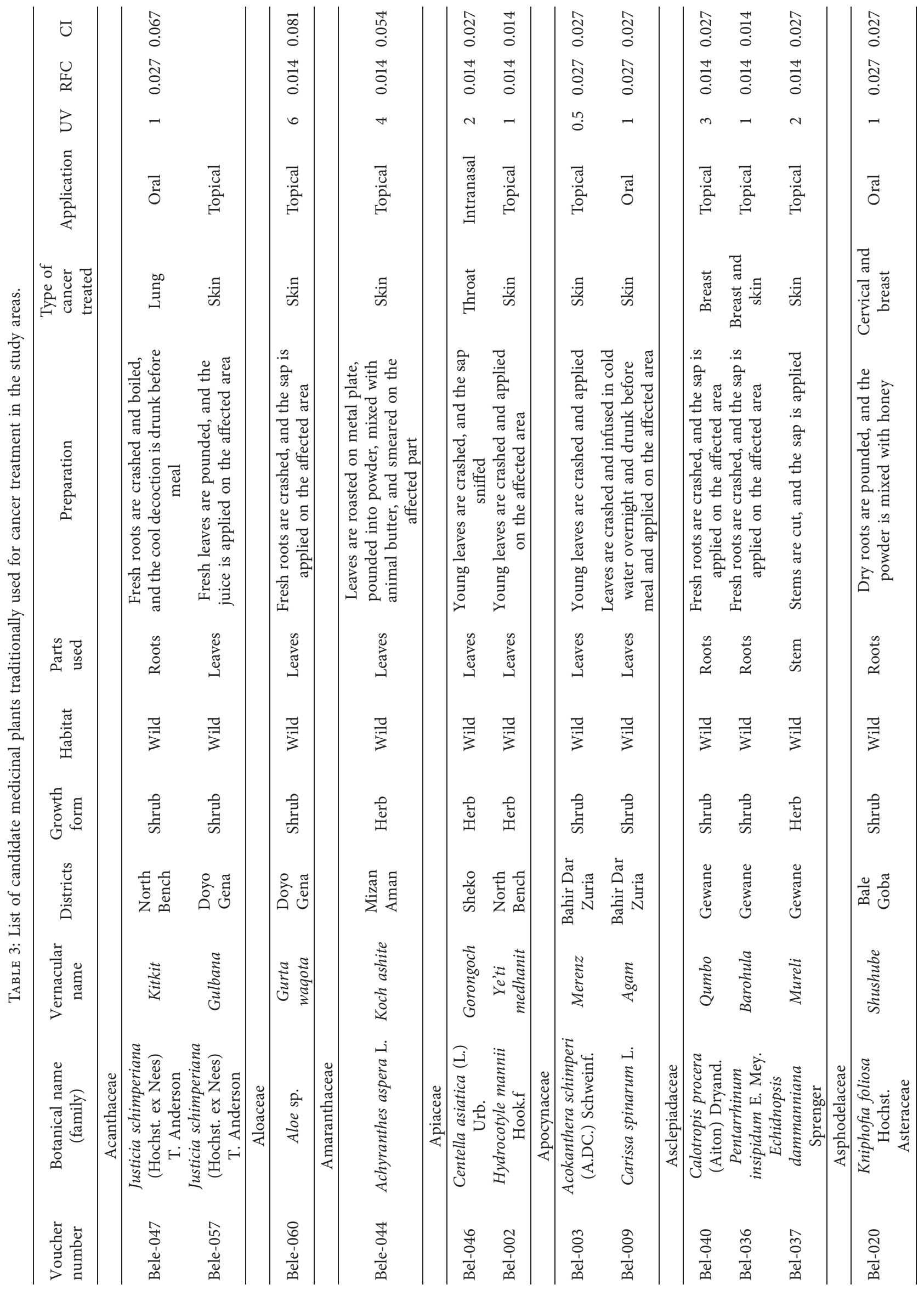




\begin{tabular}{|c|c|c|c|c|c|c|c|c|c|c|c|c|c|c|c|c|c|c|}
\hline$\vec{v}$ & 苨 & $\begin{array}{l}\hat{\sigma} \\
\text { Oे. }\end{array}$ & $\begin{array}{l}\vec{H} \\
\dot{0} \\
0\end{array}$ & $\stackrel{\vec{H}}{\stackrel{H}{0}}$ & $\begin{array}{l}\sharp \\
\stackrel{2}{0} \\
0 \\
0\end{array}$ & $\begin{array}{l}\vec{H} \\
\stackrel{0}{0} \\
0\end{array}$ & $\begin{array}{l}\hat{0} \\
\text { Oे } \\
0\end{array}$ & $\begin{array}{l}\vec{\infty} \\
\stackrel{0}{0} \\
\dot{0}\end{array}$ & & & $\begin{array}{l}\vec{H} \\
0 \\
0\end{array}$ & & $\begin{array}{l}\widehat{O} \\
\text { ờ } \\
\dot{0}\end{array}$ & $\begin{array}{l}\vec{F} \\
\dot{0}\end{array}$ & \multicolumn{4}{|c|}{$\begin{array}{l}0 \\
0\end{array}$} \\
\hline 吕 & 菅 & $\begin{array}{l}\vec{H} \\
\stackrel{0}{0}\end{array}$ & $\begin{array}{l}\ddot{H} \\
\dot{0}\end{array}$ & $\begin{array}{l}\ddot{H} \\
\dot{0} \\
\dot{0}\end{array}$ & $\begin{array}{l}\text { ते } \\
\text { Oें }\end{array}$ & $\begin{array}{l}\stackrel{H}{0} \\
\stackrel{0}{0}\end{array}$ & $\begin{array}{l}+ \\
\dot{0} \\
0 \\
0\end{array}$ & Fे & & & 范 & & $\begin{array}{l}+ \\
\\
0\end{array}$ & ते & & 30 & & \\
\hline 3 & $\begin{array}{l}\hat{0} \\
0\end{array}$ & N & - & - & $\stackrel{n}{-}$ & - & $\sim$ & $\stackrel{m}{-}$ & & & $m$ & & N & $\stackrel{n}{\rightarrow}$ & & $\begin{array}{l}\stackrel{n}{n} \\
0\end{array}$ & & \\
\hline 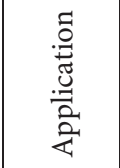 & 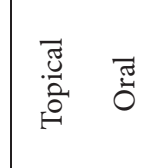 & $\overline{0}$ & ప్రే & चٓ & 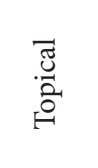 & 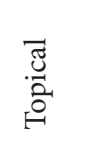 & 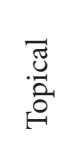 & 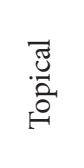 & 荧 & & 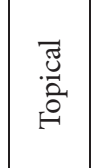 & & 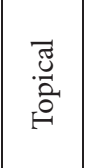 & 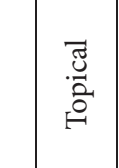 & & 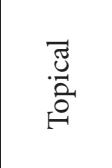 & $\frac{\text { त्र }}{\stackrel{a}{0}}$ & \\
\hline 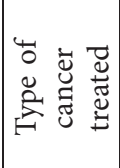 & 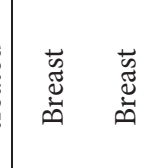 & 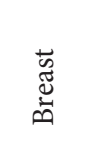 & 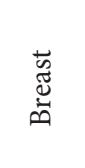 & 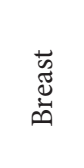 & 寻 & $\frac{\mathrm{g}}{\mathrm{s}}$ & $\frac{\tilde{z}}{\omega}$ & $\frac{\text { 专 }}{\omega}$ & 毒 & & 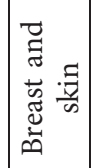 & & $\frac{\text { 春 }}{\omega}$ & 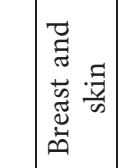 & & 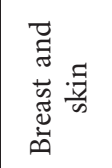 & 灵 & 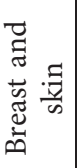 \\
\hline 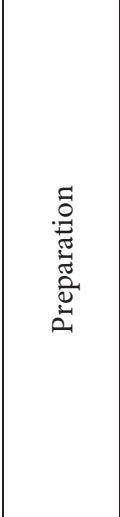 & 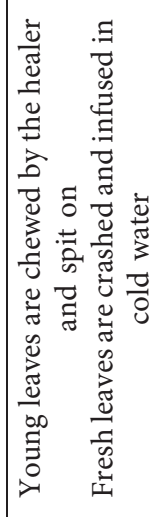 & 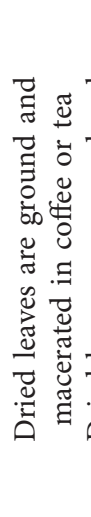 & 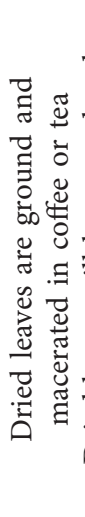 & 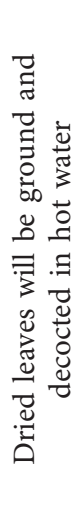 & 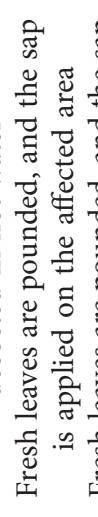 & 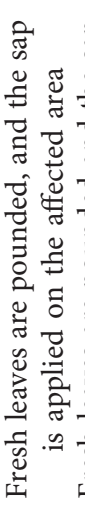 & 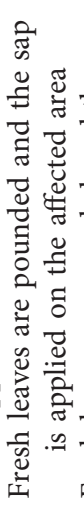 & 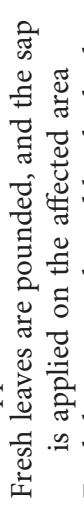 & 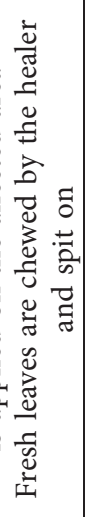 & & 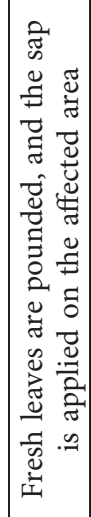 & & 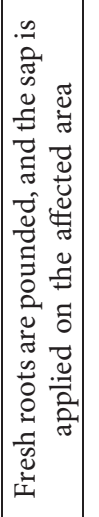 & 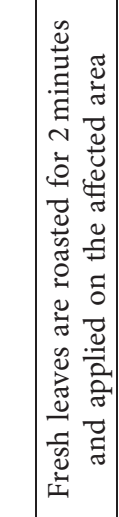 & & 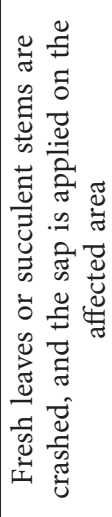 & 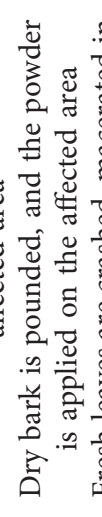 & 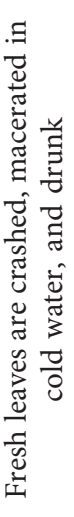 \\
\hline 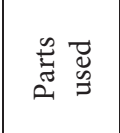 & 总 & 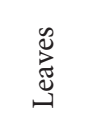 & $\stackrel{\mathscr{\Xi}}{\stackrel{\Xi}{\Theta}}$ & 岕 & 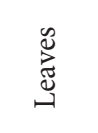 & 亗 & $\stackrel{\mathscr{\Xi}}{\leftrightarrows}$ & 预 & 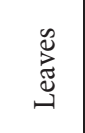 & & 芯 & & वै & 岕 & & 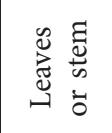 & 竞 & 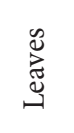 \\
\hline 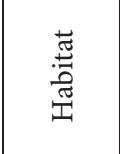 & 尝 & 국 & 曾 & 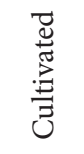 & 旁 & $\frac{7}{3}$ & 胥 & 旁 & 奈 & & 旁 & & 营 & $\frac{7}{3}$ & & $\frac{7}{3}$ & 굴 & 曾 \\
\hline 营茧 & $\begin{array}{l}\vec{E} \\
\text { 节 }\end{array}$ & 离 & 总 & $\stackrel{\dddot{\Perp}}{\sharp}$ & 离 & 㤩 & 言 & 言 & 苛 & & 莀 & & 总 & 紊 & & $\underset{\Xi}{\sharp}$ & $\stackrel{\Xi}{\sharp ~}$ & 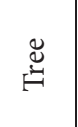 \\
\hline 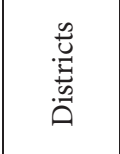 & 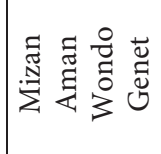 & $\frac{\pi}{0} \cdot \frac{\pi}{g}$ & 응 & 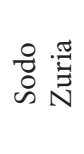 & 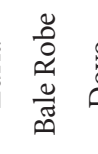 & $\begin{array}{ll}\circ & \pi \\
0 & 0 \\
0 & 0 \\
0 & 0\end{array}$ & & & 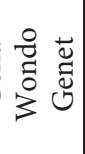 & & 苋 & & 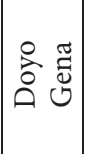 & 尝 & & 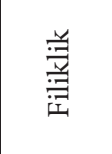 & ○芯芯 & \\
\hline $\begin{array}{l}\text { 节 } \\
\text { 节 } \\
\text { 苛 }\end{array}$ & 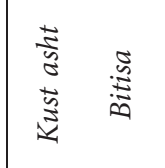 & $\frac{a}{5}$ & $\frac{5}{5}$ & 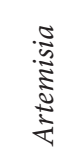 & $\begin{array}{l}\text { है } \\
\text { है } \\
\text { है }\end{array}$ & $\begin{array}{l}\frac{5}{0} \\
\frac{0}{2} \\
\text { के }\end{array}$ & $\frac{\sqrt{4}}{\sqrt[3]{2}}$ & 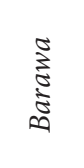 & $: \bar{\Xi}$ & & 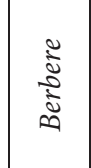 & & 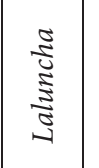 & $\begin{array}{l}0 \\
\text { 童 } \\
\frac{5}{4}\end{array}$ & & 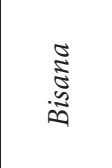 & 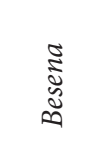 & $\begin{array}{l}8 \\
\frac{5}{\tilde{y}} \\
\vdots \\
\Sigma\end{array}$ \\
\hline 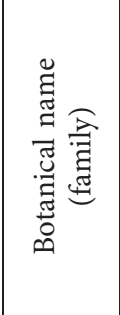 & 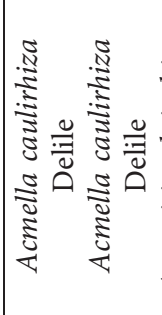 & 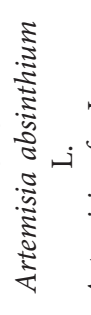 & 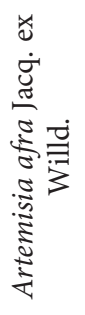 & 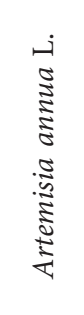 & 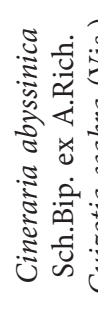 & 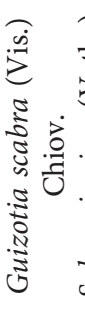 & 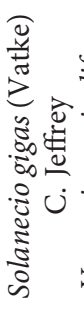 & 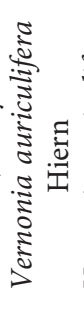 & 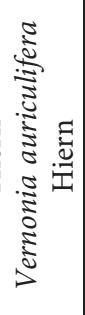 & 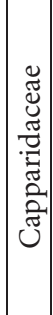 & 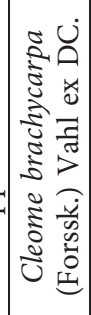 & 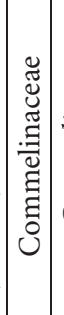 & 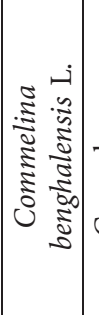 & 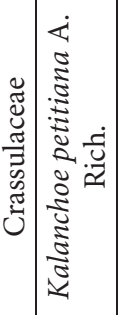 & 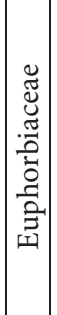 & 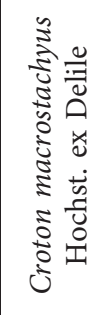 & 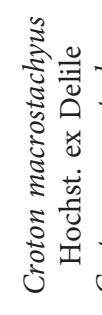 & 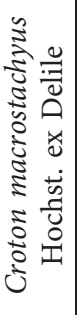 \\
\hline 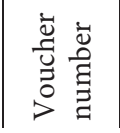 & $\begin{array}{l}\stackrel{9}{0} \\
\stackrel{i}{i} \\
\infty\end{array}$ & $\begin{array}{l}\infty \\
\infty \\
i \\
i \\
\infty\end{array}$ & సิ & $\begin{array}{l}\overrightarrow{\tilde{o}} \\
\frac{1}{\tilde{\omega}} \\
\vec{n}\end{array}$ & 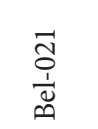 & $\begin{array}{l}\infty \\
\infty \\
0 \\
i \\
i \\
\infty\end{array}$ & $\begin{array}{l}\stackrel{+1}{\infty} \\
\stackrel{1}{\Phi} \\
\infty\end{array}$ & 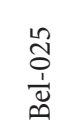 & 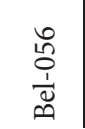 & & 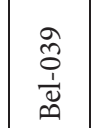 & & $\begin{array}{l}\stackrel{2}{1} \\
\frac{1}{\tilde{D}} \\
\infty\end{array}$ & $\begin{array}{l}\overrightarrow{0} \\
\frac{1}{\Phi} \\
\infty\end{array}$ & & 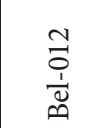 & $\begin{array}{l}\stackrel{n}{\infty} \\
\stackrel{0}{1} \\
\stackrel{i}{\Phi}\end{array}$ & $\begin{array}{l}\infty \\
\stackrel{0}{0} \\
\frac{1}{N} \\
\curvearrowleft\end{array}$ \\
\hline
\end{tabular}




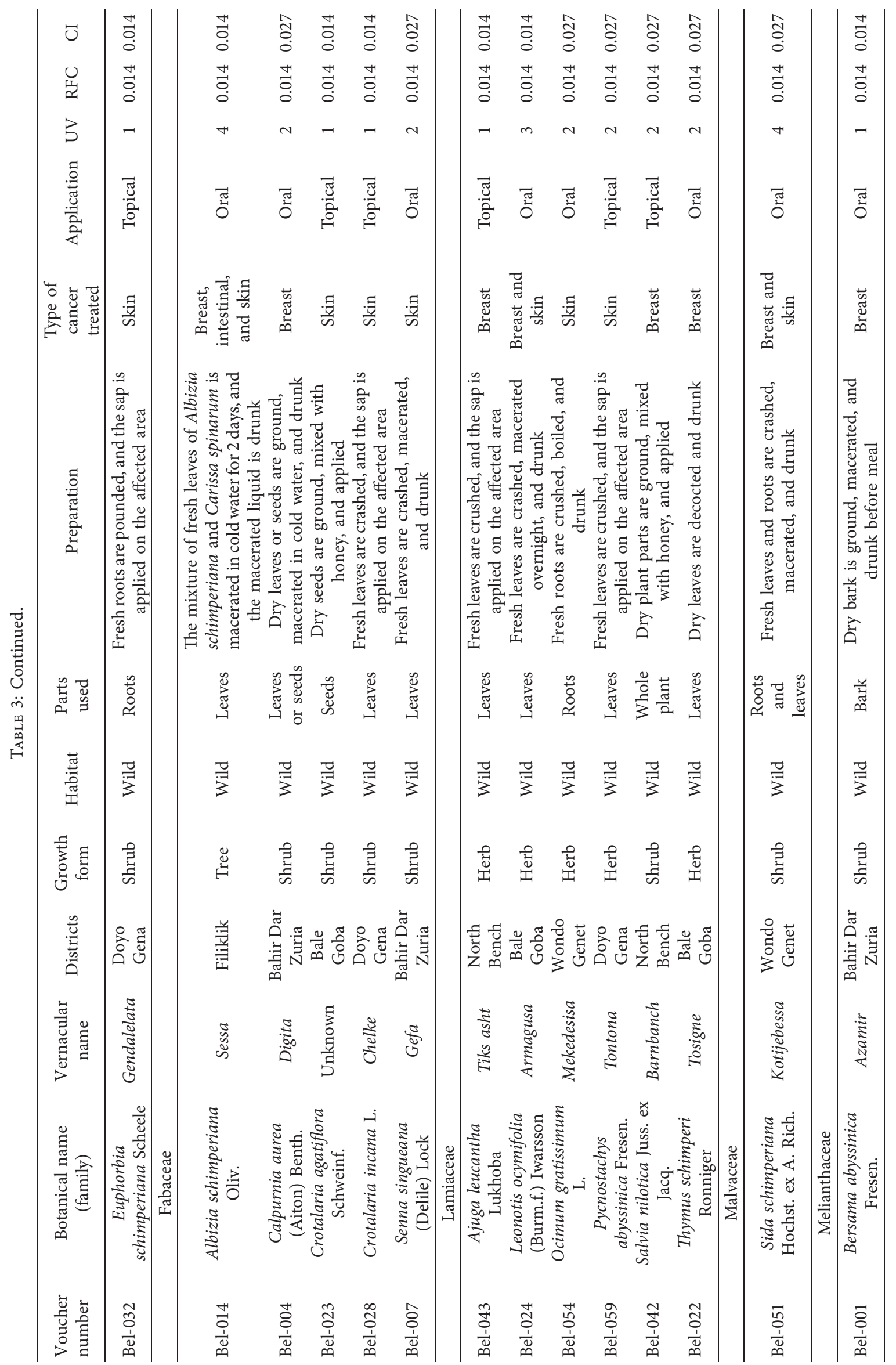




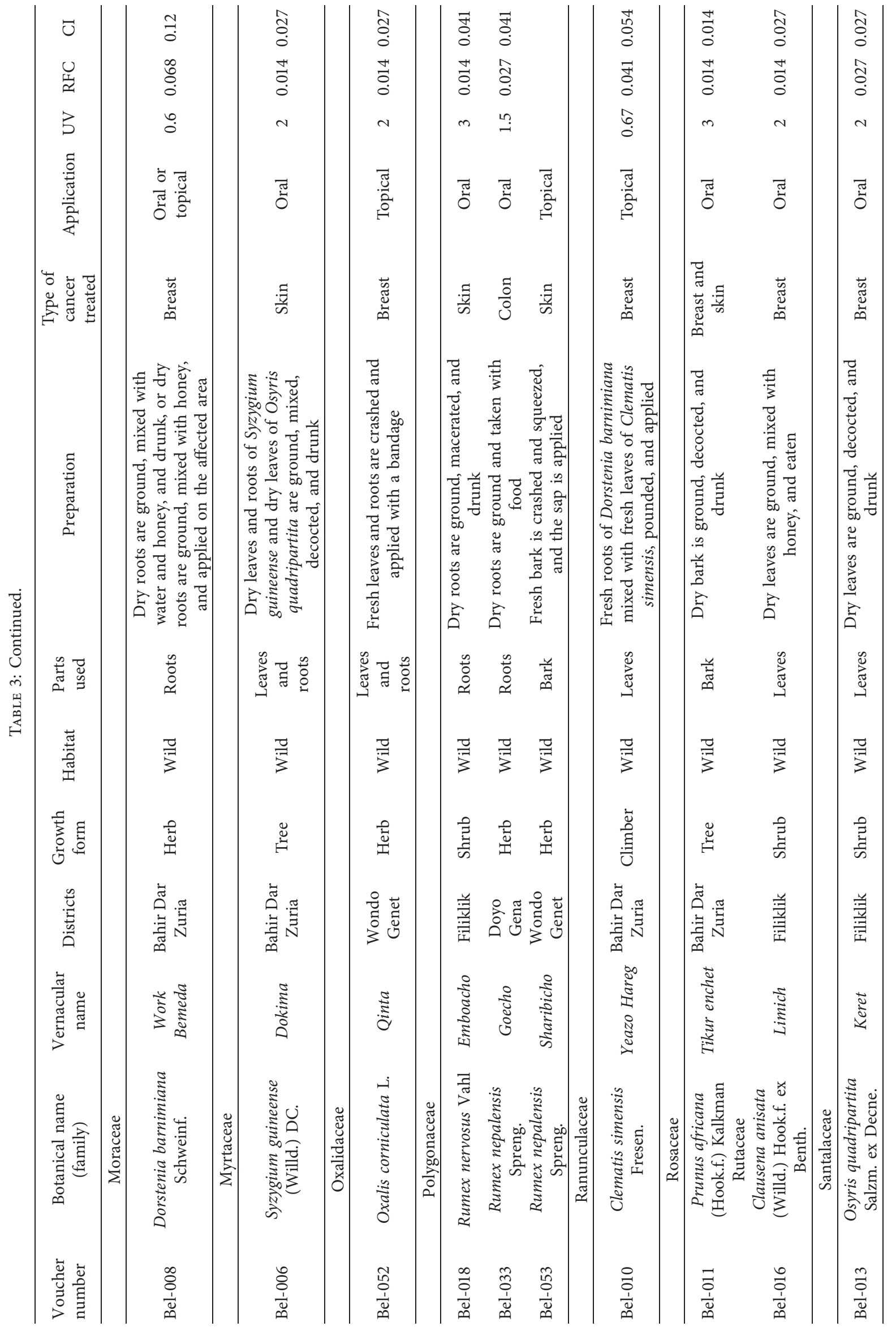




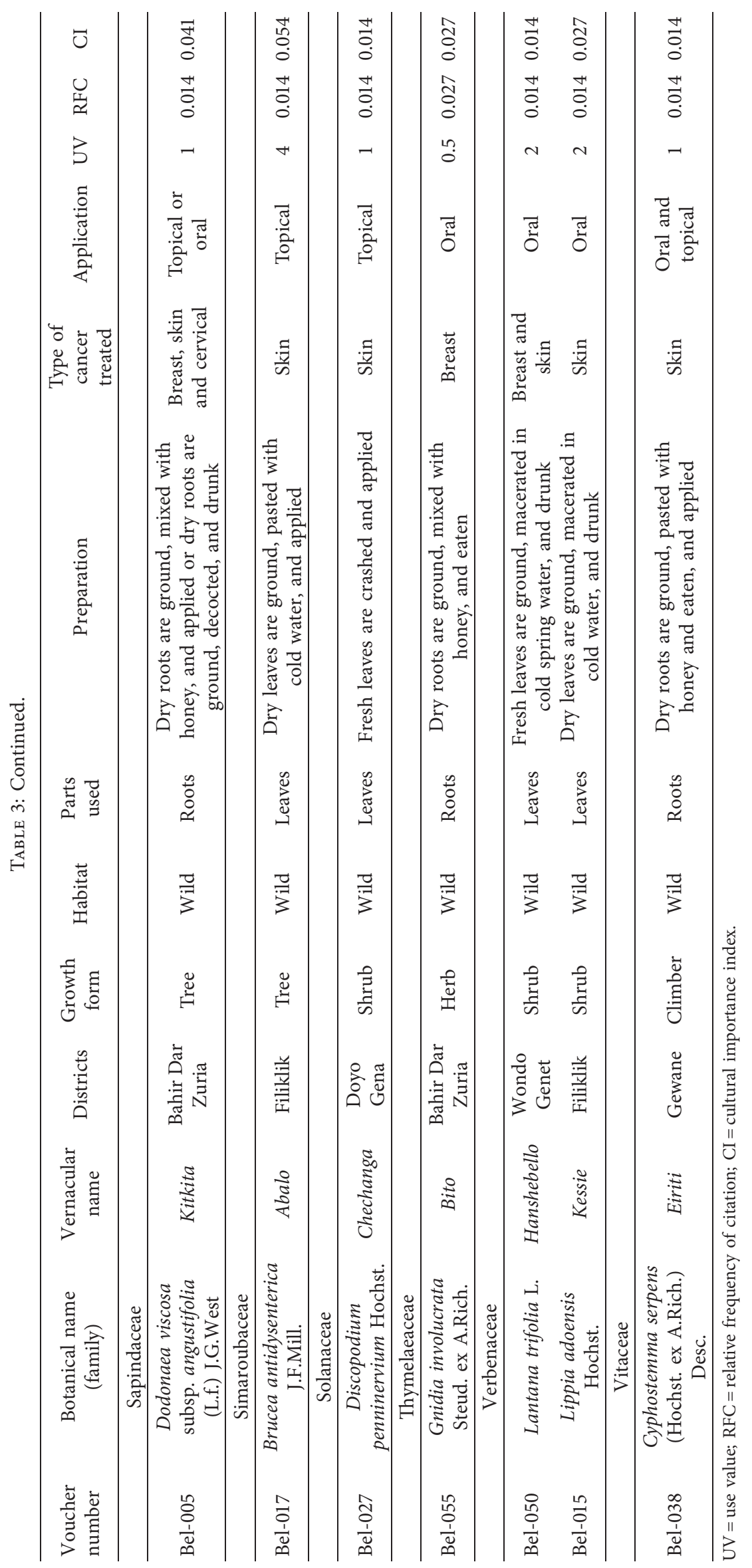




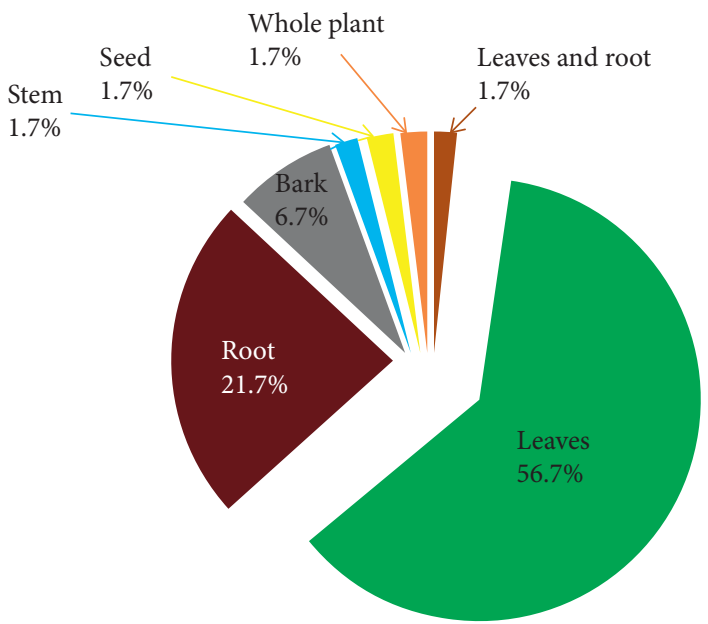

Figure 3: Frequency of plant parts used for the preparation of medicinal remedy.

TABLE 4: Cross-reference of cancer treatment candidate plant species collected from the study areas with the published literature.

\begin{tabular}{lc}
\hline Botanical name (family) & Biological activity/chemical constituents \\
\hline & Saponins, alkaloids, terpenoids and flavonoids [32] \\
In vitro cytotoxicity [33]; in vitro antioxidant \\
activity on DPPH assay [34]; in vivo suppression of \\
Justicia schimperiana \\
(Hochst. ex Nees) T. \\
Anderson (Acanthaceae) \\
in the 4-day suppressive test [32]; and in vivo \\
hepatoprotective activity in mice intoxicated with \\
CCL $_{4}$ [35]
\end{tabular}

Anthrones and chromones [51], pyrones, coumarins, alkaloids, glycoproteins, naphthalenes, and flavonoids [52]

Aloe sp. (Aloaceae)

7-O-methylaloeresin showed in vitro antioxidant activity in DPPH assay [51], and methanol and ethanol extract showed in vivo parasitaemia suppression on Plasmodium berghei-infected mice in the 4-day suppressive test $[53,54]$
Achyranthes aspera L. (Amaranthaceae)
Phytosteroids, polyphenols, and saponins [60] Methanol extracts have showed in vivo wound healing activity [61]
Illnesses/symptoms claimed to be treated traditionally

Wound [15, 21]; rabies [15, 18-20, 36-39]; jaundice $[15,16,21,23,38,40]$; gonorrhea $[17,36,39]$; liver cirrhosis $[18,26]$; seizure $[19,41]$; stomach ache

$[15,25,38]$; helminths $[15,42,43]$; skin burn/lesion $[23,44]$; arthritis $[21,23]$; hepatitis $[45,46]$; evil eye $[15,46]$; dysentery $[15,21]$; malaria $[36,39]$; common cold, asthma, and headache [36, 39, 47]; otitis [48]; toothache [49]; and rheumatism [50]

Wound [21, 55]; eye disease [21, 46, 48, 56]; snake bite $[21,48,56]$; malaria $[20,21,44,48,54]$; easing labour [44]; tropical ulcer, colon cleaner, and gallstone [48]; amoeba, abdominal pain, impotence, and urine retention [21]; dandruff $[46,56]$, hemorrhoids and hepatitis B [46]; ascariasis [21]; diabetes [54]; asthma [55]; foot strain [57, 58]; wart and anthrax [20]; external injury [59]; and liver swelling, splenomegaly, and skin inflammation [56]

Bleeding [21, 24, 26, 62-64]; retained placenta [21, 62]; stomach ache and external swelling [17]; rhesus factor incompatibility in pregnancy [40, 55]; epistaxis [19]; hepatitis and evil eye [24]; tonsillitis [21, 57]; snake bite and paralysis [21]; dysentery [59]; herpes zoster [26]; anthrax [21, 49]; nasal infection and ophthalmic infection [64]; excessive menstruation and tape worm infection [15]; and gonorrhea [65]

Terpenoids (triterpenes, asiaticoside, centelloside, madecassoside, brahmoside, brahminoside (saponin glycosides), asiaticentoic acid, centellic acid, centoic acid, madecassic acid, terminolic acid, betulic acid, $\beta$-caryophyllene, trans- $\beta$-farnesene and germacrene $\mathrm{D}$ (sesquiterpenes), $\alpha$-pinene, and $\beta$-pinene $[66,67]$

Centella asiatica (L.) Urb. (Apiaceae)
Genital infection and lymphadenitis [63]; topical swelling [26, 70]; gastritis, headache, and evil eye [70]; bleeding [40]; wound [24]; abdominal ache [71]; meningitis [72]; and tinea corporis [47]
Methanol extract inhibited the proliferation of human gastric adenocarcinoma (MK-1), human uterine carcinoma (HeLa), and murine melanoma

(B16F10) cells in vitro [68]; aqueous extracts induced apoptosis in colonic crypts and exerted chemopreventive effect on colon tumorigenesis in male F344 rats [69] 
TABLE 4: Continued.

\begin{tabular}{lcc}
\hline Botanical name (family) & Biological activity/chemical constituents & Illnesses/symptoms claimed to be treated traditionally \\
\hline & $\begin{array}{c}\text { In vitro cytotoxicity [73]; in vitro antiviral activity } \\
\text { against coxsackie B3, influenza A, and herpes }\end{array}$ & \\
& simplex type1 virus [74]; in vitro antimicrobial & Wound $[16,44,77,78] ;$ hepatitis [15, 16, 22, 44]; \\
Acokanthera schimperi & activity against Staphylococcus aureus, & gonorrhea [19, 25]; evil eye [62]; bone fracture [24]; \\
(A.DC.) Schweinf. & Pseudomonas aeruginosa, Trichophyton & hemorrhoids [44]; scabies [21]; malaria and tonsillitis \\
(Apocynaceae) & mentagrophytes [75]; and in vivo parasitaemia & [48, 56]; psychiatric disease [55]; and skin diseases [65] \\
& suppression in Plasmodium berghei-infected mice &
\end{tabular}

[76]

Carissa spinarum L. (Apocynaceae)
In vitro antioxidant activity on DPPH assay and in antiproliferative activity [79]
Throat cancer $[23,80]$; evil eye

$[16,21,24,49,62,70,72,81]$; snake bite $[23,80]$; gonorrhea [20, 65]; stomach ache [20, 70]; impotence and headache [20]; tonsillitis [17, 56, 70]; wound and febrile illness [16]; bleeding after delivery [44]; muscle cramps [49]; toothache [47]; and premature ejaculation [56]

Latex contains phytochemicals such as alkaloids, sterols, fatty acids, starches, sugars, oils, tannins, resins, and gums, and enzymatic proteins such as proteases, chitenases, lipases, peptidases, esterase,

peroxidases, papain, hevein, and lectins [82]

Calotropis procera (Aiton) Dryand. (Asclepiadaceae)

In vivo hepatoprotective [83]; hypoglycemic effect [84]; strong anti-implantation (antifertility) [85]; crude latex showed antioxidant and antiapoptotic activities against the toxicity of 4-nonylphenol [86]
Wound [16, 21, 81]; hemorrhoids [16, 19, 44]; wart $[16,57]$; snake bite $[23,87]$; kidney stone, tuberculosis, and scabies [16]; swelling [58]; skin rash [21, 49]; tinea capitis [21]

Pentarrhinum insipidum E. Mey. (Asclepiadaceae) No previous reports

Echidnopsis dammanniana Sprenger (Asclepiadaceae)

Kniphofia foliosa Hochst. (Asphodelaceae)

No previous reports
Snake bite [56]

2-Acetyl-1-hydroxy-8-methoxy-3-

methylnaphthalene, 10-(chrysophanol-7'-yl)-10-

(६)-hydroxychrysophanol-9-anthrone,

chryslandicin, knipholone, and chrysophanol [88]

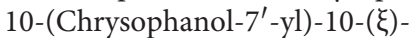

hydroxychrysophanol-9-anthrone showed in vitro antiplasmodial activity against chloroquinesensitive 3D7 strain of Plasmodium falciparum and knipholone selectively inhibited leukotriene metabolism in in vitro a human blood assay [88]; knipholone anthrone showed in vitro cytotoxicity [89] and antioxidant activity on DPPH assay [90]

Unsaturated alkylamides like spilanthol and Nisobutylnona-2E,4E-dien-8ynamide [91] In vitro antiplasmodial activity [92]

Acmella caulirhiza Delile (Asteraceae)
Camphor, davanone, ethyl (E)-cinnamate, (E)nerolidol, and chamazulene [93]

Essential oils showed in vitro antiparasitic effects Artemisia absinthium L. (Asteraceae)

Artemisia afra Jacq. ex Willd. (Asteraceae) against promastigote and axenic amastigote forms of Leishmania donovani and Leishmania aethiopica and in vitro cytotoxicity on THP-1 (human leukaemia) cell lines [93]; and in vitro cytotoxicity on human leukaemia cell lines [94]

Epoxylinalol and dihydrocostunolide [94]; camphor, davanone, bornyl acetate, 4-terpineol, and chamazulene [95]

In vitro cytotoxicity on human leukaemia cell lines [73]; and in vitro antioxidant effect on DPPH assay [95]
Swelling [15]; tonsillitis [20, 63]; and toothache [40, 87] No previous reports

Stomach ache [18, 42]; evil eye [16, 17, 62]; headache $[42,77]$; eye disease, tinea capitis infection, hematuria, and stabbing pain [77]; antifertility agent [33]; malaria $[42,62]$; ascariasis [18]; epilepsy and febrile illness $[46,65]$ 
TABLE 4: Continued.

\begin{tabular}{lll}
\hline Botanical name (family) & Biological activity/chemical constituents & Illnesses/symptoms claimed to be treated traditionally \\
\hline $\begin{array}{l}\text { In vitro inhibition of immune mediators of } \\
\text { angiogenesis [96]; the sesquiterpene (Z)-7- }\end{array}$ &
\end{tabular}
acetoxy-methyl-11-methyl-3-methylene-dodeca1,6,10-triene showed moderate cytotoxic activities against the human tumor cell lines of HO8910 Artemisia annua L. (ovary), 95-D (lung), QGY (liver), and HeLa (Asteraceae) (cervix) by MTT assay and induced apoptosis on No previous reports

95-D tumor cells [97]; artemisinin and quercetagetin $6,7,3^{\prime}, 4^{\prime}$-tetramethyl ether showed significant cytotoxicity against P-388, A-549, HT29, MCF-7, and KB tumor cells [98]

Cineraria abyssinica Sch.Bip. In vitro radical scavenging activity on DPPH assay Cineraria abyssinica Sch.Bip. [99]; flavonoidal glycoside (rutin) showed in vitro
ex A.Rich. (Asteraceae) antibacterial activity [100]

No previous reports

Guizotia scabra (Vis.) Chiov. In vitro cytotoxicity on human leukaemia cell lines Wound [20]; epilepsy [40]; and ectoparasite infestation (Asteraceae) [73], and in vitro antiviral activity [101] [47]

Solanecio gigas (Vatke) C. Jeffrey (Asteraceae)

In vitro antiviral activity against human immunodeficiency virus type 1 and type 2 cytotoxicity on human T-lymphocytic MT-4 cell lines [102]

Vernonia auriculifera Hiern Tannins, flavonoids, terpenoids, and saponins (Asteraceae) [103]

Cleome brachycarpa

(Forssk.) Vahl ex DC. (Capparidaceae)

Commelina benghalensis $\mathrm{L}$. (Commelinaceae)

Phlobatannins, carbohydrates, tannins, glycosides,

volatile oils, resins, balsams, flavonoids, and saponins [104]

Ethanol extract showed in vivo sedative and anxiolytic activity [105]

Polyphenols, alkaloids, flavonoids, tannins, saponins, and steroids [106]

Kalanchoe petitiana A. Rich. In vitro antimicrobial activity against Escherichia (Crassulaceae) coli, Pseudomonas aeruginosa, and Staphylococcus aureus [75]; and in vivo wound healing activity [106]

Croton macrostachyus

Hochst. ex Delile

(Euphorbiaceae)
Ethanol extract showed in vitro antioxidant activity on DPPH assay [79]
Skin diseases [62]; retained placenta [40]; hepatitis [64]; evil eye [15]

Toothache [72]; snake bite [42]; skin cut [47]

No previous reports 
TABLE 4: Continued.

\begin{tabular}{|c|c|c|}
\hline Botanical name (family) & Biological activity/chemical constituents & Illnesses/symptoms claimed to be treated traditionally \\
\hline $\begin{array}{l}\text { Calpurnia aurea (Aiton) } \\
\text { Benth. (Fabaceae) }\end{array}$ & $\begin{array}{l}3 \beta, 4 \alpha, 13 \alpha \text {-Trihydroxylupanine, calpaurine, } \\
\text { lupinine, and epilupinine calpurmenine and } \\
\text { calpurmenine pyrrolecarboxylic acid ester, 13- } \\
\text { hydroxylupanine, its tiglate and pyrrolecarboxylic } \\
\text { acid esters (calpumine), virgiline and virgiline } \\
\text { pyrrolecarboxylic acid ester [113]; } 4 \beta \text {-hydroxy- } \\
13 \alpha-O-\left(2^{\prime} \text {-pyrrolylcarbonyl)-lupanine (digittine) }\right. \\
\text { and } 4 \beta, 13 \alpha \text {-dihydroxylupanine [114]; alkaloids, } \\
\text { tannins, flavonoids, and saponins [35] } \\
\text { Methanol extract showed in vitro antimicrobial } \\
\text { activity against Staphylococcus aureus, Escherichia } \\
\text { coli, and Pseudomonas aeruginosa [75] and type } 1 \\
\text { and type } 2 \text { human immunodeficiency virus and } \\
\text { showed cytotoxicity on human T-lymphocytic } \\
\text { MT-4 cell lines [102]; methanol and } \\
\text { dichloromethane crude extracts showed in vitro } \\
\text { cytotoxicity on human leukaemia cells [73]; and } \\
\text { ethanol extracts showed in vitro antioxidant } \\
\text { activity on DPPH assay [79] }\end{array}$ & $\begin{array}{l}\text { Tumor }[22,26,80] \text {; stomach ache }[21,62,70,81] \text {; } \\
\text { wound and skin infection [62]; Gonorrhoea and } \\
\text { syphilis [16], amoebiasis }[16,80] \text {; ascariasis and gastric } \\
\text { ulcer [23]; diarrhea }[21,38,70] \text {; scabies and pubic hair } \\
\text { louse [40]; diabetes mellitus and hypertension [19]; } \\
\text { herpes zoster, hemorrhoids and tinea capitis [21]; and } \\
\text { swelling and tuberculosis [58] }\end{array}$ \\
\hline $\begin{array}{l}\text { Crotalaria agatiflora } \\
\text { Schweinf. (Fabaceae) }\end{array}$ & $\begin{array}{c}\text { Methanol and dichloromethane crude extracts } \\
\text { showed in vitro cytotoxicity on human leukaemia } \\
\text { cells [73] }\end{array}$ & \\
\hline $\begin{array}{l}\text { Crotalaria incana L. } \\
\text { (Fabaceae) }\end{array}$ & $\begin{array}{c}\text { Dihydrosenecionine isomer, nemorensine isomer, } \\
\text { integerrimine and anacrotine [115] } \\
\text { Methanol and dichloromethane crude extracts } \\
\text { showed in vitro cytotoxicity on human leukaemia } \\
\text { cell lines [73] }\end{array}$ & No previous reports \\
\hline $\begin{array}{l}\text { Senna singueana (Delile) } \\
\text { Lock (Fabaceae) }\end{array}$ & $\begin{array}{c}\text { Methanol extracts showed in vitro antioxidant } \\
\text { activity on DPPH assay [116] }\end{array}$ & $\begin{array}{c}\text { Stomach ache }[58,62,70] \text {; wound and swellings }[62] \text {; } \\
\text { teeth infection and sprain }[58]\end{array}$ \\
\hline $\begin{array}{l}\text { Ajuga leucantha Lukhoba } \\
\text { (Lamiaceae) }\end{array}$ & No previous reports & Diarrhea $[70]$ \\
\hline
\end{tabular}

Leonotis ocymifolia (Burm.f.) Methanol and dichloromethane crude extracts Iwarsson (Lamiaceae)

showed in vitro cytotoxicity on human leukaemia cells [73]

Essential oil contains constitutes $\gamma$-terpinene,

Ocimum gratissimum L. (Lamiaceae)

$\beta$-phellandrene, limonene, and thymol and showed in vivo antiplasmodial activity against Plasmodium berghei infection [117]

Pycnostachys abyssinica

Fresen. (Lamiaceae)

No previous reports
Ascariasis [62], febrile illness [16, 62]; eye disease [16]; headache and neck ulcer [55]; and snake bite [15]
Allergy reaction $[18,20]$; rheumatism, headache and eye disease [18]; febrile illness and general malaise [40]; sun stroke [24]; malaria [44]

Eye disease [18, 47]; ascariasis and wound [18]; diarrhea, stomach ache, amoebiasis, stomach bloating, and food poisoning [70]; headache [63]

Essential oil contains germacrene D, guaiol, and trans-caryophyllene as major constituents and Salvia nilotica Juss. ex Jacq. showed activity against both Gram-positive and (Lamiaceae) Gram-negative pathogenic bacteria; the oil also showed in vitro antioxidant activity on DPPH assay [118]

Thymus schimperi Ronniger (Lamiaceae)

Phenol and flavonoid compounds, and aqueous methanol extract showed in vitro radical scavenging ability, iron reducing power, and total antioxidant capacity [119]

Sida schimperiana Hochst. ex A. Rich. (Malvaceae)
No previous reports
Tonsillitis and constipation [62]; herpes simplex $[18,38]$; wound [40]; lymphadenitis [63]; and hemorrhoids and diarrhea [65]

Diabetes [62]; hypertension [18, 40]; tonsillitis [18]; toothache $[18,21]$; abdominal pain [21]; and cough $[38,55]$

"Shotelaye" (hydrops fetalis) [21, 22]; cough and fever [62]; diarrhea [18]; wound [25, 62]; bleeding and evil eye [24]; glandular disease and rabies [40]; amoebic dysentery, and liver disease [65]; paralysis [21]; epilepsy [43] 
TABLE 4: Continued.

\begin{tabular}{ccc}
\hline Botanical name (family) & Biological activity/chemical constituents & Illnesses/symptoms claimed to be treated traditionally \\
\hline & Flavonol glycosides isoquercetrin, hyperoside, \\
quercetin-3-O-arabinopyranoside, kaempferol-3- & O-arabinopyranoside, xanthone glycoside,
\end{tabular}

$\mathrm{O}$-arabinopyranoside, xanthone glycoside, mangiferin [115]

Bersama abyssinica Fresen. Ethanol water extracts showed in vitro antioxidant (Melianthaceae)

activity on DPPH assay and antiproliferative activity on human liver carcinoma cell line and normal human fetal lung cells [79]; methanol extract showed in vitro antioxidant activity on DPPH assay [115], and antiviral activity against type 1 human immunodeficiency virus [102]

Dorstenia barnimiana Phytochemical screening showed the presence of Schweinf. (Moraceae) coumarins [34]

Methanol and dichloromethane crude extracts Syzygium guineense (Willd.) DC. (Myrtaceae) showed in vitro cytotoxicity on human leukaemia cells [73] and antimicrobial activity [120]

Oxalis corniculata $\mathrm{L}$. In vivo antitumor activity against Ehrlich ascites (Oxalidaceae) carcinoma on mice [121]

Rumex nervosus Vahl (Polygonaceae)

Alkaloids, flavonoids, terpenoids, tannins, glycosides, and volatile oils [122]
Tumor, dysentery and roundworms [107, 109]; ascariasis [15, 38, 81, 109]; wound [20]; stomach ache [17]; snake bite and liver diseases [70]; tonsillitis [72]; bronchitis and febrile illness [42, 43]

Cancer [26]; hepatitis, syphilis and rabies [25, 26]; skin cancer, dysentery, wart and fever [25]; pulmonary tuberculosis, leprosy, and stomach illness [22]

Stomach ache [17-19, 23]; diarrhea [15, 18, 19, 24],

kidney infection, liver cirrhosis, and tonsillitis [18];

syphilis [23, 80]; malaria, hemorrhoid, internal worms, snake bite, and gonorrhea [65]

Wound [17]; arthritis [63]; tape worm infection [21]

Breast cancer, gastritis, and snake bite [16]; wart

$[15,22]$; hepatitis $[49,55]$; skin rash $[16,21]$; bleeding

$[15,40,81,109]$; wound $[40,49,55,62,109,110]$;

scabies and acne vulgaris [62]; ascariasis and herpes simplex [21]; stomach ache and dysentery [22];

diarrhea [49]; eye problems and round worm [55]

Anthraquinones, naphthalenes, tannins, stilbenoids [123]

Ethanol water extracts showed in vitro antiproliferative activity on human liver

Rumex nepalensis Spreng. (Polygonaceae)

Clematis simensis Fresen. (Ranunculaceae)

carcinoma cell line and on normal human fetal lung cells and antioxidant activity on DPPH assay [79], and methanol and dichloromethane crude extracts showed in vitro cytotoxicity on human leukaemia cells [73]

Triterpenoids, saponins, alkaloids, polyphenols, and unsaturated sterols [120]

In vivo anti-inflammatory and antinociceptive activities [124]

Prunus africana (Hook.f.)

Kalkman (Rosaceae)

No previous reports
Wound, ascariasis, abdominal bleeding, gastric ulcer, and hemorrhage $[23,80]$; gastritis [18]; stomach

problems [108]; leishmaniasis [25]; abdominal cramp and ear infection [63]; tonsillitis $[18,25]$

Carbazole alkaloids, peptide derivatives, sitosterol

Clausena anisata (Willd.) and stigmasterol [125]

Hook.f. ex Benth. (Rutaceae)

Methanol and dichloromethane crude extracts

showed in vitro cytotoxicity on human leukaemia cells [73]

Alkaloids, phenols, terpenoids, tannins, saponins,

Osyris quadripartita Salzm. ex Decne. (Santalaceae) and flavonoids [126]
Methanol extracts showed in vitro antimicrobial activity against Escherichia coli, Pseudomonas aeruginosa, Staphylococcus aureus, Candida albicans, and Trichophyton mentagrophytes [11]; in vitro inhibition of $\mathrm{NO}$ production and cytotoxicity against MCF-7 and NCI-H460 cell lines [127]
Cancer [62]; anaphylactic shock, evil eye, and epilepsy [18]; eczema [40]; toothache [46] 
TABLE 4: Continued.

\begin{tabular}{lcc}
\hline Botanical name (family) & Biological activity/chemical constituents & Illnesses/symptoms claimed to be treated traditionally \\
\hline & $\begin{array}{c}\text { Alkaloids, terpenoids, saponins, tannins, sugars, } \\
\text { phenolics, and flavonoids [128] }\end{array}$ & \\
$\begin{array}{l}\text { Dethanol extracts showed in vivo nonsensitizer } \\
\text { angustifolia (L.f.) J.G.West } \\
\text { (Sapindaceae) }\end{array}$ & $\begin{array}{c}\text { effect in mice using the mouse ear swelling test } \\
\text { method [129], in vitro antiviral effect against type 1 } \\
\text { human immunodeficiency virus [102], and in vitro } \\
\text { free radical scavenging activity on DPPH assay } \\
\text { [128] }\end{array}$ & Malaria [57] \\
\hline $\begin{array}{l}\text { Brucea antidysenterica } \\
\text { J.F.Mill. (Simaroubaceae) }\end{array}$ & $\begin{array}{c}\text { Flavonoids, amino acids, and vitamin C [130] } \\
\text { In vitro antiplasmodial activity against } \\
\text { Plasmodium berghei infection [131] }\end{array}$ & Cancer/tumor [107]; wart [24]; rabies [18, 62]; leprosy \\
\hline [62]
\end{tabular}

$5 \alpha, 17 \beta$-Dihydroxy- $6 \alpha, 7 \alpha$-epoxy-1-oxowitha-2,24-

dienolide, withanone, and withanolide A [132],

5,6-epoxy-16-oxygenated withanolides, jaborosalactone-L, and 17-epiacnistin-A

$[133,134] ; 6 \alpha, 7 \alpha$-epoxy-1-oxo- $5 \alpha, 12 \alpha, 17 \alpha$ -

trihydroxywitha-2,24-dienolide and a coloratane sesquiterpene, $7 \alpha, 11 \alpha$-dihydroxy-4(13),8-

coloratadien-12,11-olide, withanone, $5 \alpha, 17 \beta$ -

dihydroxy- $6 \alpha, 7 \alpha$-epoxy-1-oxowitha-2,24-

dienolide, $7 \alpha, 11 \alpha$-dihydroxy-8-drimen-12,11olide, withasomnine, and (E,Z)-9-

Discopodium penninervium

Hochst. (Solanaceae)

hydroxyoctadeca-10,12-dienoic acid [135]

Jaborosalactone-L showed cytotoxicity only to the murine macrophage cell line, RAW 264.7, but the $16 \alpha$-oxygenated withanolides exhibited cytotoxicity to both human (COR-L23 and ECV 304) and murine (L929 and RAW 264.7) carcinoma cell lines with $\mathrm{IC}_{50}$ values ranging from 1.2 to $150 \mu \mathrm{M}$ [136]. $6 \alpha, 7 \alpha$-Epoxy-1-oxo$5 \alpha, 12 \alpha, 17 \alpha$-trihydroxy-witha-2,24-dienolide inhibited COX-2 and LTB4 formation; $7 \alpha, 11 \alpha$ dihydroxy-4(13),8-coloratadien-12,11-olide and withasomnine inhibited $\mathrm{LTB}_{4}$ biosynthesis but showed minor inhibition of COX-1 and COX-2 [135]

Gnidia involucrata Steud. ex A.Rich. (Thymelaeaceae) Flavonoids and glycosides [137] Flavone glycosides (scutellarein-7-O- $\beta$-Dapiofuranoside and apigenin-7-O- $\beta$-D-

Lantana trifolia L. (Verbenaceae) apiofuranosyl-(1 $\longrightarrow 2)-\beta$-D-apiofuranoside) and the flavone celtidifoline $(5,6,40,50$-tetrahydroxy7,30-dimethoxyflavone) $[138,139]$

Limonene, perillaldehyde, piperitenone, and 2methyl-6-methylene-2,7-octadien-4-one [140], sesquiterpene hydrocarbon (germacrene D) [141] Methanol extract showed in vitro cytotoxicity on Lippia adoensis Hochst. (Verbenaceae) human leukaemia cell lines [73], and antimicrobial activity against Staphylococcus aureus, Escherichia coli, and Pseudomonas aeruginosa [75]; water extracts showed in vivo protection/relieve against acetic acid induced writhing in mice model [142]

Eczema, fungal infections, common cold, and cough [62]; intestine swelling [18]; gastrointestinal disorder [40]; abdominal irritation and acute stomach illness [46]
Skin detoxification [62]; and liver disease [70]
Ascariasis, evil eye, anthrax, intestinal helminths, and gland swelling [18]
Cyphostemma serpens

(Hochst. ex A.Rich.) Desc. (Vitaceae) highest ICF value (0.42) recorded for "other ailments" category, in this study, suggests that informants are in agreement with the use of particular plant species to treat

\section{No previous reports}

ailments in this category. The lowest ICF value (0) obtained was for lung, colon, cervical, and throat cancer categories. This might be due to the cultural and ecological differences 
TABLE 5: Informants consensus factor for different ailment categories.

\begin{tabular}{|c|c|c|c|c|c|c|}
\hline No. & Category & No. of species & $\%$ of all species & No. of use reports & $\%$ of all use reports & ICF \\
\hline 1 & Skin & 25 & 30.5 & 32 & 26.5 & 0.23 \\
\hline 2 & Breast & 20 & 24.4 & 29 & 23.9 & 0.32 \\
\hline 3 & Cervical & 1 & 1.22 & 1 & 0.83 & 0 \\
\hline 4 & Colon & 1 & 1.22 & 1 & 0.83 & 0 \\
\hline 5 & Lung & 1 & 1.22 & 1 & 0.83 & 0 \\
\hline 6 & Throat & 1 & 1.22 & 1 & 0.83 & 0 \\
\hline \multirow[t]{2}{*}{7} & Other disease & 33 & 40.2 & 56 & 46.3 & 0.42 \\
\hline & Total & $82^{*}$ & & 121 & & \\
\hline
\end{tabular}

*Each plant species may be listed in several categories.

TABLE 6: Summary of stats for relative frequency of citation (RFC) and cultural importance index (CI).

\begin{tabular}{lcccc}
\hline & Mean & Standard deviation & Minimum & Maximum \\
\hline UV & 1.8 & 1.1 & 0.5 & 6 \\
RFC & 0.02 & 0.015 & 0.014 & 0.1 \\
CI & 0.034 & 0.027 & 0.014 & 0.16 \\
\hline
\end{tabular}

Association between RFC and CI by using Pearson correlation method

\begin{tabular}{lccc} 
& UV & RFC & CI \\
\hline UV & 1 & & \\
RFC & $-0.36^{*}$ & 1 & \\
CI & 0.003 & $0.858^{* *}$ & 1 \\
\hline
\end{tabular}

${ }^{*}$ Correlation is significant at 0.05 level. ${ }^{* *}$ Correlation is significant at 0.001 level.

of the study sites and the difficulty to pinpoint the physical symptoms of lung, colon, cervical, and throat cancer as compared to the breast and skin cancer.

The present study also revealed that RFC and CI values of some reported species are similar. However, there is a distinct difference in species ranking using each index. $C$. macrostachyus is placed in the first position according to both RFC and CI index. This could be due to the fact that this species is mentioned by many informants and is the most recognized plant in most study areas. Furthermore, CI value of C. macrostachyus is also high, suggesting the diversified use of the plant. $V$. auriculifera and $C$. simensis ranked next to C. macrostachyus, according to RFC index. On the other hand, D. barnimiana and Aloe spp. ranked $2^{\text {nd }}$ and $3^{\text {rd }}$ by CI index. It has been suggested that UV value is a good measure of use diversity, than the number of citations [144]. In agreement with this, UV value in our study is driven by species with greatest number of use rather than those cited by more informants. The Pearson correlation coefficient of -0.36 , between RFC and UV, shows significant negative association between the local importance of each medicinal plant and relative importance of use of plants. This result is in contrast to previous studies that reported a significant positive correlation between RFC and UV $[145,146]$. On the other hand, there is a significant positive correlation between RFC and CI $\left(r^{2}=0.74, p<0.001\right)$ implying that their pattern matches across species. The species with larger RFC value usually have higher CI, such as Croton macrostachyus and Vernonia auriculifera.

Leaves and roots are the most commonly used plant parts in the preparation of remedies in the study districts.
Similarly, other ethnobotanical studies conducted in different parts of Ethiopia also reported that leaves are the dominant plant part followed by root [16-18, 20]. The preference towards leaves may be because leaves are the main photosynthetic organs in plants and the primary reservoirs for secondary metabolites with medicinal values [36]. In contrary to other ethnobotanical studies [17, 18], where the common use of concoctions and oral route were reported, in the current study majority of the reported remedies are prepared from a single plant species and applied topically.

Comparative analysis of this study with other ethnobotanical surveys of plants used traditionally in treating and managing cancer in Ethiopia [18], Kenya [147], Cameroon [37], Nigeria [19, 38], South Africa [39], and Bangladesh [148] revealed some similarities in the plants cited in these surveys. Of the 30 plant species cited to be used in Ethiopia [18], 7 species are identified in our study: Bersama abyssinica Fresen., Brucea antidysenterica JF. Mill., Calpurnia aurea (Ait.) Benth. Dodonaea angustifolia L.f., Dorstenia barnimiana Schweinf, Kalanchoe petitiana A. Rich., and Prunus africana (Hook. f) Kalkm.

Although herbal remedies are believed by the general public to be safe [46], some research findings suggested otherwise. For instance, traditionally used Thai anticancer plants Ganoderma lucidum (Fr.) Karst., Houttuynia cordata Thunb., and Saussurea involucrata Matsum. \& Koidz. were reported to cause side effects such as headache, insomnia, constipation, and diarrhea [62]. Similarly, side effects such as vomiting, diarrhea, and skin necrosis, associated with the use of traditional herbal remedies, were reported in this and other ethnobotanical studies conducted in Ethiopia $[149,150]$. Few side effects reported in this study, as compared to other ethnobotanical studies conducted in Ethiopia, could be attributed to the frequent use of the topical route of administration. Nevertheless, considering the probability of underreporting adverse effects, extensive toxicological investigations should be conducted to protect the public.

In vitro cytotoxicity and antioxidant properties of some of the plants reported in our study have also been studied. Among these plants, potent cytotoxic activity was reported for knipholone anthrone isolated from Kniphofia foliosa, with $\mathrm{IC}_{50}$ value that ranges between $0.9 \pm 0.1$ and $3.3 \pm 0.4 \mu \mathrm{g} / \mathrm{mL}$ [89]. Similarly, Nibret and Wink reported the cytotoxic activity of the crude extract of Acokanthera 
schimperi with $\mathrm{IC}_{50}$ value of $7.1 \mu \mathrm{g} / \mathrm{mL}$ [73]. Studies conducted on the leaves of Cineraria abyssinica [100], bark of Senna singueana [116], and bark and leaves of Rumex nepalensis [79] also revealed potent radical scavenging activity of these plants.

\section{Conclusion}

The present study showed that traditional healers in eleven districts of Ethiopia use different medicinal plants to manage cancer-like symptoms. Frequency of citation value ranked Croton macrostachyus Del., Clematis simensis Fresen., Dorstenia barnimiana Schweinf, Vernonia auriculifera Hiern, and Acmella caulirhiza Del. as most cited plant species in study areas. Hence, based on these findings, we are currently evaluating the in vitro antiproliferative activities of reported medicinal plant species with a higher frequency of citation against human breast adenocarcinoma (MCF-7), human uterine cervical adenocarcinoma (SiSo), human lung carcinoma (A-427), and human bladder cancer (RT-4) cell lines using crystal violate assay. However, considering the rapid disappearance of the traditional knowledge of medicinal plants and an urgent need for new anticancer agents, additional studies have to be conducted to document and scientifically validate traditionally used Ethiopian anticancer plants.

\section{Data Availability}

The authors declare that all data supporting the finding of this study are included in this article and its supplementary information files.

\section{Ethical Approval}

Ethical approval was obtained from Addis Ababa University, College of Health Sciences Ethics Review Board (Ref no. $\mathrm{ERB} / \mathrm{SOP} / 126 / 12 / 2015)$.

\section{Consent}

Each participant consented before the interview.

\section{Conflicts of Interest}

The authors declare that they have no conflicts of interest.

\section{Authors' Contributions}

A.B., E.E., and K.A. jointly conceived the study. S.T. conducted the ethnobotanical study and taxonomical identification. S.T., A.B., E.E., T.G., and K.A. enriched the draft manuscript for its intellectual content. All authors read and approved the final manuscript.

\section{Acknowledgments}

The authors wish to thank all traditional healers, individuals, data collectors, and local administrative authorities in all study districts that made this survey possible. This research was supported by the thematic research grant from the Addis Ababa University (grant number: TR/35/2015).

\section{References}

[1] J. Zhang, S. S. Späth, S. L. Marjani, W. Zhang, and X. Pan, "Characterization of cancer genomic heterogeneity by nextgeneration sequencing advances precision medicine in cancer treatment," Precision Clinical Medicine, vol. 1, no. 1, pp. 29-48, 2018.

[2] H. H. Heng, J. B. Stevens, S. W. Bremer, K. J. Ye, G. Liu, and C. J. Ye, "The evolutionary mechanism of cancer," Journal of Cellular Biochemistry, vol. 109, no. 6, pp. 1072-1084, 2014.

[3] G. Finak, N. Bertos, F. Pepin et al., "Stromal gene expression predicts clinical outcome in breast cancer," Nature Medicine, vol. 14, no. 5, pp. 518-527, 2008.

[4] H.-C. Wu, D.-K. Chang, and C.-T. Huang, "Targeted therapy for cancer," Journal of Molecular Cancer, vol. 2, no. 2, pp. 57-66, 2010.

[5] J. A. Roth, F. Fossella, R. Komaki et al., "A randomized trial comparing perioperative chemotherapy and surgery with surgery alone in resectable stage IIIA non-small-cell lung cancer," JNCI Journal of the National Cancer Institute, vol. 86, no. 9, pp. 673-680, 1994.

[6] K. Gregory and A. Tutt, "Managing side-effects of cancer therapy," in Cancer in Primary Care, M. E. Gore and D. Russell, Eds., pp. 47-88, Taylor and Francis Publishers, London, UK, 2003.

[7] M. M. Gottesman, "Mechanisms of cancer drug resistance," Annual Review of Medicine, vol. 53, no. 1, pp. 615-627, 2002.

[8] G. M. Cragg, D. J. Newman, and K. M. Snader, "Natural products in drug discovery and development," Journal of Natural Products, vol. 60, no. 1, pp. 52-60, 1997.

[9] A. Harvey, "Medicines from nature: are natural products still relevant to drug discovery?" Trends in Pharmacological Sciences, vol. 20, no. 5, pp. 196-198, 1999.

[10] K. Hostettmann, A. Marston, K. Ndjoko, and J.-L. Wolfender, "The potential of African plants as a source of drugs," Current Organic Chemistry, vol. 4, no. 10, pp. 973-1010, 2000.

[11] S. Taddese, K. Asres, and T. Gebre-Mariam, "In vitro antimicrobial activities of some selected topically applied medicinal plants of Ethiopia," Ethiopian Pharmaceutical Journal, vol. 21, pp. 39-46, 2003.

[12] S. D. E. Kelbessa, Z. Woldu, and S. Edwards, "Some threatened endemic plants of Ethiopia," in The Status of Some Plants in Parts of Tropical Africa, Z. A. S. Edwards, Ed., pp. 35-55, Addis Ababa University, Addis Ababa, Ethiopia, 1992.

[13] K. D. Kassaye, A. Amberbir, B. Getachew, and Y. Mussema, "A historical overview of traditional medicine practices and policy in Ethiopia," Ethiopian Journal of Health Development, vol. 20, no. 2, pp. 127-134, 2007.

[14] N. Tuasha, B. Petros, and Z. Asfaw, "Medicinal plants used by traditional healers to treat malignancies and other human ailments in Dalle District, Sidama Zone, Ethiopia," Journal of Ethnobiology and Ethnomedicine, vol. 14, no. 1, p. 15, 2018.

[15] G. Chekole, Z. Asfaw, and E. Kelbessa, "Ethnobotanical study of medicinal plants in the environs of Tara-gedam and Amba remnant forests of Libo Kemkem district, northwest Ethiopia," Journal of Ethnobiology and Ethnomedicine, vol. 11, no. 1, p. 4, 2015.

[16] S. Araya, B. Abera, and M. Giday, "Study of plants traditionally used in public and animal health management in 
Seharti Samre district, Southern Tigray, Ethiopia," Journal of Ethnobiology and Ethnomedicine, vol. 11, no. 1, p. 22, 2015.

[17] R. Regassa, "Assessment of indigenous knowledge of medicinal plant practice and mode of service delivery in Hawassa city, southern Ethiopia," Journal of Medicinal Plants Research, vol. 7, no. 9, pp. 517-535, 2013.

[18] M. Agize, S. Demissew, and Z. Asfaw, "Ethnobotany of medicinal plants in Loma and Gena bosa districts (woredas) of dawro zone, southern Ethiopia," Topclass Journal of Herbal Medicine, vol. 2, no. 9, pp. 194-212, 2012.

[19] S. Suleman and T. Alemu, "A survey on utilization of ethnomedicinal plants in Nekemte town, East Wellega (Oromia), Ethiopia," Journal of Herbs, Spices \& Medicinal Plants, vol. 18, no. 1, pp. 34-57, 2012.

[20] B. Abera, "Medicinal plants used in traditional medicine by Oromo people, Ghimbi district, Southwest Ethiopia," Journal of Ethnobiology and Ethnomedicine, vol. 10, no. 1, p. 40, 2014.

[21] A. Teklay, B. Abera, and M. Giday, "An ethnobotanical study of medicinal plants used in Kilte Awulaelo district, Tigray region of Ethiopia," Journal of Ethnobiology and Ethnomedicine, vol. 9, no. 1, p. 65, 2013.

[22] T. Teklehaymanot, M. Giday, G. Medhin, and Y. Mekonnen, "Knowledge and use of medicinal plants by people around Debre Libanos monastery in Ethiopia," Journal of Ethnopharmacology, vol. 111, no. 2, pp. 271-283, 2007.

[23] M. Ragunathan and S. M. Abay, "Ethnomedicinal survey of folk drugs used in Bahirdar Zuria district, Northwestern Ethiopia," Indian Journal of Traditional Knowledge, vol. 8, pp. 281-284, 2009.

[24] T. Mekuanent, A. Zebene, and Z. Solomon, "Ethnobotanical study of medicinal plants in Chilga district, Northwestern Ethiopia," Journal of Natural Remedies, vol. 15, no. 2, pp. 88-112, 2015.

[25] T. Teklehaymanot, "Ethnobotanical study of knowledge and medicinal plants use by the people in Dek Island in Ethiopia," Journal of Ethnopharmacology, vol. 124, no. 1, pp. 69-78, 2009.

[26] T. Teklehaymanot and M. Giday, "Ethnobotanical study of medicinal plants used by people in Zegie Peninsula, northwestern Ethiopia," Journal of Ethnobiology and Ethnomedicine, vol. 3, no. 1, p. 12, 2007.

[27] CSA, "The 2007 population and housing census of Ethiopia: administrative report," Central Statistical Agency, Addis Ababa, Ethiopia, 2012.

[28] I. Friis, S. Demissew, and P. Van Breugel, "Atlas of the potential vegetation of Ethiopia," Det Kongelige Danske Videnskabernes Selskab, Copenhagen, Denmark, 2010.

[29] J. Tardío and M. Pardo-de-Santayana, "Cultural importance indices: a comparative analysis based on the useful wild plants of southern cantabria (northern Spain)1," Economic Botany, vol. 62, no. 1, pp. 24-39, 2008.

[30] O. Phillips and A. H. Gentry, "The useful plants of Tambopata, Peru: I. Statistical hypotheses tests with a new quantitative technique," Economic Botany, vol. 47, no. 1, pp. 15-32, 1993.

[31] M. Heinrich, A. Ankli, B. Frei, C. Weimann, and O. Sticher, "Medicinal plants in Mexico: healers' consensus and cultural importance," Social Science \& Medicine, vol. 47, no. 11, pp. 1859-1871, 1998.

[32] J. Abdela, E. Engidawork, and W. Shibeshi, "In vivo antimalarial activity of solvent fractions of the leaves of justicia schimperiana hochst. Ex Nees against Plasmodium berghei in Mice," Ethiopian Pharmaceutical Journal, vol. 30, no. 2, pp. 95-108, 2014.

[33] B. Desta, "Ethiopian traditional herbal drugs-part III: antifertility activity of 70 medicinal plants," Journal of Ethnopharmacology, vol. 44, no. 3, pp. 199-209, 1994.

[34] R. A. Mothana, R. Gruenert, P. Bednarski, and U. Lindequist, "Evaluation of the in vitro anticancer, antimicrobial and antioxidant activities of some Yemeni plants used in folk medicine," Die Pharmazie-An International Journal of Pharmaceutical Sciences, vol. 64, no. 4, pp. 260-268, 2009.

[35] S. Umer, K. Asres, and C. Veeresham, "Hepatoprotective activities of two Ethiopian medicinal plants," Pharmaceutical Biology, vol. 48, no. 4, pp. 461-468, 2010.

[36] H. Agisho, M. Osie, and T. Lambore, "Traditional medicinal plants utilization, management and threats in Hadiya Zone, Ethiopia," Journal of Medicinal Plants Research, vol. 2, no. 2, pp. 94-108, 2014.

[37] A. Enyew, Z. Asfaw, E. Kelbessa, and R. Nagappan, "Status of medico-cultural commercial plants at Fiche town market, Ethiopia," International Journal of Pharmaceuticals and Health Care Research, vol. 1, no. 4, pp. 227-236, 2013.

[38] Z. Kassa, Z. Asfaw, and S. Demissew, "Ethnobotanical study of medicinal plants used by the local people in tulu korma and its surrounding areas of ejere district, western shewa zone of oromia regional state, Ethiopia," Journal of Medicinal Plants Studies, vol. 4, no. 2, pp. 24-47, 2016.

[39] S. Zerabruk and G. Yirga, "Traditional knowledge of medicinal plants in Gindeberet district, Western Ethiopia," South African Journal of Botany, vol. 78, pp. 165-169, 2012.

[40] A. Kefalew, Z. Asfaw, and E. Kelbessa, "Ethnobotany of medicinal plants in ada'a district, east shewa zone of oromia regional state, Ethiopia," Journal of Ethnobiology and Ethnomedicine, vol. 11, no. 1, p. 25, 2015.

[41] T. T. Ayele, M. B. Regasa, and D. A. Delesa, "Antibacterial and antagonistic activity of selected traditional medicinal plants and herbs from East Wollega zone against clinical isolated human pathogens," Science, Technology and Arts Research Journal, vol. 4, no. 3, pp. 175-179, 2016.

[42] G. Bekele and P. R. Reddy, "Ethnobotanical study of medicinal plants used to treat human ailments by Guji Oromo tribes in Abaya District, Borana, Oromia, Ethiopia," Universal Journal of Plant Science, vol. 3, no. 1, pp. 1-8, 2015.

[43] F. Mesfin, S. Demissew, and T. Teklehaymanot, "An ethnobotanical study of medicinal plants in Wonago Woreda, SNNPR, Ethiopia," Journal of Ethnobiology and Ethnomedicine, vol. 5, no. 1, p. 28, 2009.

[44] M. Giday, T. Teklehaymanot, A. Animut, and Y. Mekonnen, "Medicinal plants of the shinasha, agew-awi and Amhara peoples in northwest Ethiopia," Journal of Ethnopharmacology, vol. 110, no. 3, pp. 516-525, 2007.

[45] M. A. Eshete, E. Kelbessa, and G. Dalle, "Ethnobotanical study of medicinal plants in guji agro-pastoralists, blue Hora district of Borana zone, Oromia region, Ethiopia," Journal of Medicinal Plants Studies, vol. 4, no. 2, pp. 170-184, 2016.

[46] H. Yineger, E. Kelbessa, T. Bekele, and E. Lulekal, "Plants used in traditional management of human ailments at Bale mountains national park, southeastern Ethiopia," Journal of Medicinal Plants Research, vol. 2, no. 6, pp. 132-153, 2008.

[47] M. Megersa, Z. Asfaw, E. Kelbessa, A. Beyene, and B. Woldeab, "An ethnobotanical study of medicinal plants in Wayu Tuka district, east Welega zone of oromia regional state, West Ethiopia," Journal of Ethnobiology and Ethnomedicine, vol. 9, no. 1, p. 68, 2013. 
[48] A. Belayneh, Z. Asfaw, S. Demissew, and N. F. Bussa, "Medicinal plants potential and use by pastoral and agropastoral communities in Erer Valley of Babile Wereda, Eastern Ethiopia," Journal of Ethnobiology and Ethnomedicine, vol. 8, no. 1, p. 42, 2012.

[49] T. Wondimu, Z. Asfaw, and E. Kelbessa, "Ethnobotanical study of medicinal plants around 'Dheeraa' town, Arsi Zone, Ethiopia," Journal of Ethnopharmacology, vol. 112, no. 1, pp. 152-161, 2007.

[50] H. Yineger, D. Yewhalaw, and D. Teketay, "Ethnomedicinal plant knowledge and practice of the Oromo ethnic group in southwestern Ethiopia," Journal of Ethnobiology and Ethnomedicine, vol. 4, no. 1, p. 11, 2008.

[51] G. Asamenew, D. Bisrat, A. Mazumder, and K. Asres, "In vitro antimicrobial and antioxidant activities of anthrone and chromone from the latex of Aloe harlana Reynolds," Phytotherapy Research, vol. 25, no. 12, pp. 1756-1760, 2011.

[52] E. Dagne, D. Bisrat, A. Viljoen, and B.-E. Van Wyk, "Chemistry of Aloe species," Current Organic Chemistry, vol. 4, no. 10, pp. 1055-1078, 2000.

[53] T. Deressa, Y. Mekonnen, and A. Animut, "In vivo antimalarial activities of Clerodendrum myricoides, Dodonea angustifolia and Aloe debrana against Plasmodium berghei," Ethiopian Journal of Health Development, vol. 241 pages, 2010.

[54] A. Mesfin, M. Giday, A. Animut, and T. Teklehaymanot, "Ethnobotanical study of antimalarial plants in Shinile district, Somali region, Ethiopia, and in vivo evaluation of selected ones against Plasmodium berghei," Journal of Ethnopharmacology, vol. 139, no. 1, pp. 221-227, 2012.

[55] E. d'Avigdor, H. Wohlmuth, Z. Asfaw, and T. Awas, "The current status of knowledge of herbal medicine and medicinal plants in Fiche, Ethiopia," Journal of Ethnobiology and Ethnomedicine, vol. 10, no. 1, p. 38, 2014.

[56] A. Belayneh and N. F. Bussa, "Ethnomedicinal plants used to treat human ailments in the prehistoric place of Harla and Dengego valleys, eastern Ethiopia," Journal of Ethnobiology and Ethnomedicine, vol. 10, no. 1, p. 18, 2014.

[57] G. Yirga, "Assessment of indigenous knowledge of medicinal plants in central zone of Tigray, northern Ethiopia," African Journal of Plant Science, vol. 4, no. 1, pp. 6-11, 2010.

[58] G. Zenebe, M. Zerihun, and Z. Solomon, "An ethnobotanical study of medicinal plants in Asgede Tsimbila district, northwestern Tigray, northern Ethiopia," Ethnobotany Research and Applications, vol. 10, pp. 305-320, 2012.

[59] T. Teklehaymanot and M. Giday, "Ethnobotanical study of wild edible plants of kara and kwego semi-pastoralist people in lower omo river valley, debub omo zone, SNNPR, Ethiopia," Journal of Ethnobiology and Ethnomedicine, vol. 6, no. 1, p. 23, 2010.

[60] W. Shibeshi, E. Makonnen, L. Zerihun, and A. Debella, "Effect of Achyranthes aspera L. on fetal abortion, uterine and pituitary weights, serum lipids and hormones," African Health Sciences, vol. 6, no. 2, pp. 108-112, 2006.

[61] A. Fikru, E. Makonnen, T. Eguale, A. Debella, and G. Abie Mekonnen, "Evaluation of in vivo wound healing activity of methanol extract of Achyranthes aspera L," Journal of Ethnopharmacology, vol. 143, no. 2, pp. 469-474, 2012.

[62] A. Enyew, Z. Asfaw, E. Kelbessa, and R. Nagappan, "Ethnobotanical study of traditional medicinal plants in and around Fiche district, Central Ethiopia," Current Research Journal of Biological Sciences, vol. 6, no. 4, pp. 154-167, 2014.
[63] M. Giday, Z. Asfaw, and Z. Woldu, "Ethnomedicinal study of plants used by Sheko ethnic group of Ethiopia," Journal of Ethnopharmacology, vol. 132, no. 1, pp. 75-85, 2010.

[64] E. Lulekal, Z. Asfaw, E. Kelbessa, and P. Van Damme, "Ethnoveterinary plants of Ankober district, north Shewa zone, Amhara region, Ethiopia," Journal of Ethnobiology and Ethnomedicine, vol. 10, no. 1, p. 21, 2014.

[65] F. Mesfin, T. Seta, and A. Assefa, "An ethnobotanical study of medicinal plants in Amaro Woreda, Ethiopia," Ethnobotany Research and Applications, vol. 12, pp. 341-354, 2014.

[66] J. Barnes, L. A. Anderson, J. D. Phillipson, and C. A. Newall, Herbal Medicines, Pharmaceutical Press London, London, UK, 2007.

[67] S. S. Jamil, Q. Nizami, and M. Salam, "Centella asiatica (Linn.) Urban: a review," Natural Product Radiance, vol. 6, no. 2, pp. 158-170, 2007.

[68] M. Yoshida, M. Fuchigami, T. Nagao et al., "Antiproliferative constituents from Umbelliferae plants VII: active triterpenes and rosmarinic acid from Centella asiatica," Biological \& Pharmaceutical Bulletin, vol. 28, no. 1, pp. 173-175, 2005.

[69] P. Bunpo, K. Kataoka, H. Arimochi et al., "Inhibitory effects of Centella asiatica on azoxymethane-induced aberrant crypt focus formation and carcinogenesis in the intestines of F344 rats," Food and Chemical Toxicology, vol. 42, no. 12, pp. 1987-1997, 2004

[70] B. Kidane, T. van Andel, L. van der Maesen, and Z. Asfaw, "Use and management of traditional medicinal plants by Maale and Ari ethnic communities in southern Ethiopia," Journal of Ethnobiology and Ethnomedicine, vol. 10, no. 1, p. 46, 2014.

[71] K. Tolossa, E. Debela, S. Athanasiadou, A. Tolera, G. Ganga, and J. G. Houdijk, "Ethno-medicinal study of plants used for treatment of human and livestock ailments by traditional healers in south Omo, southern Ethiopia," Journal of Ethnobiology and Ethnomedicine, vol. 9, no. 1, p. 32, 2013.

[72] M. Giday, Z. Asfaw, and Z. Woldu, "Medicinal plants of the Meinit ethnic group of Ethiopia: an ethnobotanical study," Journal of Ethnopharmacology, vol. 124, no. 3, pp. 513-521, 2009.

[73] E. Nibret and M. Wink, "Trypanocidal and cytotoxic effects of 30 Ethiopian medicinal plants," Zeitschrift für Naturforschung C, vol. 66, no. 11-12, pp. 0541-0546, 2011.

[74] T. Gebre-Mariam, R. Neubert, P. Schmidt, P. Wutzler, and M. Schmidtke, "Antiviral activities of some Ethiopian medicinal plants used for the treatment of dermatological disorders," Journal of Ethnopharmacology, vol. 104, no. 1-2, pp. 182-187, 2006.

[75] H. Tadeg, E. Mohammed, K. Asres, and T. Gebre-Mariam, "Antimicrobial activities of some selected traditional Ethiopian medicinal plants used in the treatment of skin disorders," Journal of Ethnopharmacology, vol. 100, no. 1-2, pp. 168-175, 2005.

[76] T. Mohammed, B. Erko, and M. Giday, "Evaluation of antimalarial activity of leaves of Acokanthera schimperi and Croton macrostachyus against Plasmodium berghei in Swiss albino mice," BMC Complementary and Alternative Medicine, vol. 14, no. 1, p. 314, 2014.

[77] D. Abebe and A. Ayehu, Medicinal Plants and Enigmatic Health Practices of Northern Ethiopia, Berhanina Selam Printing Press, Addis Ababa, Ethiopia, 1993.

[78] G. Meaza, B. Tadesse, A. S. Maria, B. Piero, and Y. Gidey, "Traditional medicinal plants used by Kunama ethnic group in northern Ethiopia," Journal of Medicinal Plants Research, vol. 9, no. 15, pp. 494-509, 2015. 
[79] J. Tauchen, I. Doskocil, C. Caffi et al., "In vitro antioxidant and anti-proliferative activity of Ethiopian medicinal plant extracts," Industrial Crops and Products, vol. 74, pp. 671-679, 2015.

[80] Z. Birhanu, "Ethno-botanical survey on medicinal plants used by ethnic groups of Denbia district, north-western Ethiopia," Journal of Natural Remedies, vol. 11, no. 2, pp. 119-123, 2011.

[81] G. Seyoum and G. Zerihun, "An ethnobotanical study of medicinal plants in Debre Libanos Wereda, Central Ethiopia," African Journal of Plant Science, vol. 8, no. 7, pp. 366-379, 2014.

[82] H. Heli, M. Amani, A. A. Moosavi-Movahedi, A. Jabbari, G. Floris, and A. Mura, "Electroactive centers in Euphorbia latex and lentil seedling amine oxidases," Bioscience, Biotechnology, and Biochemistry, vol. 72, no. 1, pp. 29-36, 2008.

[83] S. R. Setty, A. A. Quereshi, A. V. Swamy et al., "Hepatoprotective activity of Calotropis procera flowers against paracetamol-induced hepatic injury in rats," Fitoterapia, vol. 78, no. 7-8, pp. 451-454, 2007.

[84] S. Roy, R. Sehgal, B. M. Padhy, and V. L. Kumar, "Antioxidant and protective effect of latex of Calotropis procera against alloxan-induced diabetes in rats," Journal of Ethnopharmacology, vol. 102, no. 3, pp. 470-473, 2005.

[85] J. V. Kamath and A. C. Rana, "Preliminary study on antifertility activity of Calotropis procera roots in female rats," Fitoterapia, vol. 73, no. 2, pp. 111-115, 2002.

[86] A. E.-D. H. Sayed, N. H. Mohamed, M. A. Ismail, W. M. Abdel-Mageed, and A. A. M. Shoreit, "Antioxidant and antiapoptotic activities of Calotropis procera latex on Catfish (Clarias gariepinus) exposed to toxic 4-nonylphenol," Ecotoxicology and Environmental Safety, vol. 128, pp. 189194, 2016.

[87] T. Flatie, T. Gedif, K. Asres, and T. Gebre-Mariam, "Ethnomedical survey of Berta ethnic group Assosa zone, Benishangul-Gumuz regional state, mid-west Ethiopia," Journal of Ethnobiology and Ethnomedicine, vol. 5, no. 1, p. 14, 2009.

[88] A. A. Wube, F. Bucar, K. Asres, S. Gibbons, L. Rattray, and S. L. Croft, "Antimalarial compounds from Kniphofia foliosa roots," Phytotherapy Research, vol. 19, no. 6, pp. 472-476, 2005.

[89] S. Habtemariam, "Knipholone anthrone from Kniphofia foliosa induces a rapid onset of necrotic cell death in cancer cells," Fitoterapia, vol. 81, no. 8, pp. 1013-1019, 2010.

[90] S. Habtemariam, "Antioxidant activity of Knipholone anthrone," Food Chemistry, vol. 102, no. 4, pp. 1042-1047, 2007.

[91] N. R. Crouch, A. Langlois, D. A. Mulholland, J. J. Nair, and P. Houghton, "A novel alkylamide from the leaves of Acmella caulirhiza (Asteraceae), a traditional surface analgesic," South African Journal of Botany, vol. 71, no. 2, pp. 228-230, 2005.

[92] B. O. Owuor, J. O. Ochanda, J. O. Kokwaro et al., "In vitro antiplasmodial activity of selected Luo and Kuria medicinal plants," Journal of Ethnopharmacology, vol. 144, no. 3, pp. 779-781, 2012.

[93] Y. Tariku, A. Hymete, A. Hailu, and J. Rohloff, "In vitro evaluation of antileishmanial activity and toxicity of essential oils of Artemisia absinthium and Echinops kebericho," Chemistry \& Biodiversity, vol. 8, no. 4, pp. 614-623, 2011.

[94] E. Nibret and M. Wink, "Volatile components of four Ethiopian Artemisia species extracts and their in vitro antitrypanosomal and cytotoxic activities," Phytomedicine, vol. 17, no. 5, pp. 369-374, 2010.

[95] M. Burits, K. Asres, and F. Bucar, "The antioxidant activity of the essential oils of Artemisia afra, Artemisia abyssinica and Juniperus procera," Phytotherapy Research, vol. 15, no. 2, pp. 103-108, 2001.

[96] X. X. Zhu, L. Yang, Y. J. Li et al., "Effects of sesquiterpene, flavonoid and coumarin types of compounds from Artemisia anпиа L. on production of mediators of angiogenesis," Pharmacological Reports, vol. 65, no. 2, pp. 410-420, 2013.

[97] D.-D. Zhai, K. Supaibulwatana, and J.-J. Zhong, "Inhibition of tumor cell proliferation and induction of apoptosis in human lung carcinoma 95-D cells by a new sesquiterpene from hairy root cultures of Artemisia annua," Phytomedicine, vol. 17, no. 11, pp. 856-861, 2010.

[98] G.-Q. Zheng, "Cytotoxic terpenoids and flavonoids from Artemisia annua," Planta Medica, vol. 60, no. 1, pp. 54-57, 1994.

[99] T. Teshome, B. Sintayehu, H. Yohannes et al., "Radical scavenging activity and preliminary phytochemical screening on aerial part extracts of Cineraria abyssinica sch. bip," Journal of Pharmacognosy and Phytochemistry, vol. 3, no. 6, pp. 239-243, 2015.

[100] B. Sintayehu, K. Asres, and Y. Raghavendra, "Radical scavenging activities of the leaf extracts and a flavonoid glycoside isolated from Cineraria abyssinica Sch. Bip. Exa. Rich," Journal of Applied Pharmaceutical Science, vol. 2, no. 4, pp. 44-49, 2012.

[101] P. Cos, N. Hermans, T. De Bruyne et al., "Further evaluation of Rwandan medicinal plant extracts for their antimicrobial and antiviral activities," Journal of Ethnopharmacology, vol. 79, no. 2, pp. 155-163, 2002.

[102] K. Asres, F. Bucar, T. Kartnig, M. Witvrouw, C. Pannecouque, and E. De Clercq, "Antiviral activity against human immunodeficiency virus type 1 (HIV-1) and type 2 (HIV-2) of ethnobotanically selected Ethiopian medicinal plants," Phytotherapy Research, vol. 15, no. 1, pp. 62-69, 2001.

[103] B. Albejo, M. Endale, B. Kibret, and M. Anza, "Phytochemical investigation and antimicrobial activity of leaves extract of Vernonia auriculifera Hiern," Journal of Pharmacy \& Pharmacognosy Research, vol. 3, no. 6, pp. 141-147, 2015.

[104] J. Ibrahim, V. C. Ajaegbu, and H. O. Egharevba, "Pharmacognostic and phytochemical analysis of Commelina benghalensis L," Ethnobotanical Leaflets, vol. 2010, no. 5, p. 7, 2010.

[105] S. Hasan, M. Hossain, R. Akter et al., "Sedative and anxiolytic effects of different fractions of the Commelina benghalensis Linn," Drug Discoveries \& Therapeutics, vol. 3, no. 5, 2009.

[106] A. Mekonnen, T. Sidamo, K. Asres, and E. Engidawork, "In vivo wound healing activity and phytochemical screening of the crude extract and various fractions of Kalanchoe petitiana A. Rich (Crassulaceae) leaves in mice," Journal of Ethnopharmacology, vol. 145, no. 2, pp. 638-646, 2013.

[107] D. Abebe, A. Debella, and K. Urga, Medicinal Plants and other Useful Plants of Ethiopia, Camerapix Publishers International, Addis Ababa, Ethiopia, 2013.

[108] R. W. Bussmann, P. Swartzinsky, A. Worede, and P. Evangelista, "Plant use in odo-bulu and demaro, Bale region, Ethiopia," Journal of Ethnobiology and Ethnomedicine, vol. 7, no. 1, p. 28, 2011.

[109] Z. Birhanu, "Traditional use of medicinal plants by the ethnic groups of Gondar Zuria district, north-western Ethiopia," Journal of Natural Remedies, vol. 13, no. 1, pp. 46-53, 2011. 
[110] Y. Limenih, S. Umer, and M. Wolde-Mariam, "Ethnobotanical study on traditional medicinal plants in Dega damot woreda, Amhara region, north Ethiopia," International Journal of Research in Pharmacy and Chemistry, vol. 5, pp. 258-273, 2015.

[111] G. Belay, Y. Tariku, T. Kebede, A. Hymete, and Y. Mekonnen, "Ethnopharmacological investigations of essential oils isolated from five Ethiopian medicinal plants against eleven pathogenic bacterial strains," Phytopharmacology, vol. 1, no. 5, pp. 133-143, 2011.

[112] H. Almehdar, H. M. Abdallah, A.-M. M. Osman, and E. A. Abdel-Sattar, "In vitro cytotoxic screening of selected Saudi medicinal plants," Journal of Natural Medicines, vol. 66, no. 2, pp. 406-412, 2012.

[113] K. Asres, W. A. Gibbons, J. D. Phillipson, and P. Mascagni, "Alkaloids of Ethiopian Calpurnia aurea subsp. aurea," Phytochemistry, vol. 25, no. 6, pp. 1443-1447, 1986.

[114] K. Asres, J. Phillipson, and P. Mascagni, "Two novel minor alkaloids from Ethiopian Calpurnia aurea sp.aurea," Planta Medica, vol. 52, no. 4, pp. 302-304, 1986.

[115] K. Asres, F. Sporer, and M. Wink, "Patterns of pyrrolizidine alkaloids in 12 Ethiopian Crotalaria species," Biochemical Systematics and Ecology, vol. 32, no. 10, pp. 915-930, 2004.

[116] M. Gebrelibanos, K. Asres, and C. Veeresham, "In vitro radical scavenging activity of the leaf and bark extracts of Senna singueana (Del). Lock," Ethiopian Pharmaceutical Journal, vol. 25, pp. 77-84, 2008.

[117] F. Tchoumbougnang, P. H. Zollo, E. Dagne, and Y. Mekonnen, "In Vivo antimalarial activity of essential oils from Cymbopogon citratus and Ocimum gratissimumon mice infected with Plasmodium berghei," Planta Medica, vol. 71, no. 1, pp. 20-23, 2005.

[118] K. Asres, H. Asfaha, A. Mazumder, and F. Bucar, "Leaf essential oils of Salvia nilotica and Salvia schimperi: their antimicrobial and antioxidant activities," Ethiopian Pharmaceutical Journal, vol. 26, no. 1, pp. 49-58, 2008.

[119] D. Engeda, B. Geremew, D. H. Gulelat, and P. V. R. H, "Antioxidant and -amylase inhibition activities in vitro of various solvent extracts of Thymus schimperi Ronniger," Journal of Medicinal Plants Research, vol. 9, no. 15, pp. 515-524, 2015.

[120] A. Geyid, D. Abebe, A. Debella et al., "Screening of some medicinal plants of Ethiopia for their anti-microbial properties and chemical profiles," Journal of Ethnopharmacology, vol. 97, no. 3, pp. 421-427, 2005.

[121] A. Kathiriya, K. Das, E. Kumar, and K. Mathai, "Evaluation of antitumor and antioxidant activity of oxalis corniculata linn: against ehrlich ascites carcinoma on mice," Iranian Journal of Cancer Prevention, vol. 34 pages, 2010.

[122] B. B. Kasimala, V. R. Anna, and U. R. Mallu, "Reverse phase liquid chromatographic method for the simultaneous estimation of antibiotic drugs: metronidazole, nalidixic acid, tinidazole and norfloxacin," Der Pharmacia Lettre, vol. 6, pp. 411-419, 2014.

[123] A. Vasas, O. Orbán-Gyapai, and J. Hohmann, "The genus rumex: review of traditional uses, phytochemistry and pharmacology," Journal of Ethnopharmacology, vol. 175, pp. 198-228, 2015.

[124] A. Tadele, K. Asres, D. Melaku, and W. Mekonnen, "In vivo anti-inflammatory and antinociceptive activities of the leaf extracts of Clematis simensis Fresen," Ethiopian Pharmaceutical Journal, vol. 27, pp. 33-41, 2010.

[125] J. Songue, E. Kouam, E. Dongo, T. Mpondo, and R. White, "Chemical constituents from stem bark and roots of
Clausena anisata," Molecules, vol. 17, no. 11, pp. 13673-13686, 2012.

[126] M. Abebaw, B. Mishra, and D. A. Gelayee, "Evaluation of anti-ulcer activity of the leaf extract of," Journal of Experimental Pharmacology, vol. 9, pp. 1-11, 2017.

[127] W. Rached, R. C. Calhelha, Â. Fernandes et al., "Phytochemical characterization and bioactive properties of Osyris quadripartita Salzm. ex Decne. leaves from Algeria," RSC Advances, vol. 6, no. 76, pp. 72768-72776, 2016.

[128] T. Riaz, M. A. Abbasi, T. Shahzadi, M. Ajaib, and K. M. Khan, "Phytochemical screening, free radical scavenging, antioxidant activity and phenolic content of Dodonaea viscosa," Journal of the Serbian Chemical Society, vol. 771 pages, 2012.

[129] K. Teshome, T. Gebre-Mariam, K. Asres, and E. Engidawork, "Toxicity studies on dermal application of plant extract of Dodonaea viscosa used in Ethiopian traditional medicine," Phytotherapy Research: An International Journal Devoted to Pharmacological and Toxicological Evaluation of Natural Product Derivatives, vol. 24, no. 1, pp. 60-69, 2010.

[130] A. Amuamuta, Z. Mekonnen, and E. Gebeyehu, "Traditional therapeutic uses and phytochemical screening of some selected indigenous medicinal plants from Northwest Ethiopia," African Journal of Pharmacology and Therapeutics, vol. 4, no. 3, 2015.

[131] A. Kefe, M. Giday, H. Mamo, and B. Erko, "Antimalarial properties of crude extracts of seeds of Brucea antidysenterica and leaves of Ocimum lamiifolium," BMC Complementary and Alternative Medicine, vol. 16, no. 1, p. 118, 2016.

[132] S. Habtemariam and A. I. Gray, "Withanolides from the roots of Discopodium penninervium," Planta Medica, vol. 64, no. 3, pp. 275-276, 1998.

[133] S. Habtemariam, A. I. Gray, and P. G. Waterman, "16-oxygenated withanolides from the leaves of Discopodium penninervium," Phytochemistry, vol. 34, no. 3, pp. 807-811, 1993.

[134] S. Habtemariam, B. W. Skelton, P. G. Waterman, and A. H. White, "17-Epiacnistin-A, a further withanolide from the leaves of discopodium p enninervium," Journal of Natural Products, vol. 63, no. 4, pp. 512-513, 2000.

[135] A. A. Wube, E.-M. Wenzig, S. Gibbons, K. Asres, R. Bauer, and F. Bucar, "Constituents of the stem bark of Discopodium penninervium and their LTB4 and COX-1 and-2 inhibitory activities," Phytochemistry, vol. 69, no. 4, pp. 982-987, 2008.

[136] S. Habtemariam, "Cytotoxicity and immunosuppressive activity of withanolides from Discopodium penninervium," Planta Medica, vol. 63, no. 1, pp. 15-17, 1997.

[137] J. Ferrari, C. Terreaux, S. Sahpaz, J. D. Msonthi, J.-L. Wolfender, and K. Hostettmann, "Benzophenone glycosides from Gnidia involucrata," Phytochemistry, vol. 54, no. 8, pp. 883-889, 2000.

[138] P. S. Imbenzi, Y.-Z. He, Z.-X. Yan, E. K. Osoro, and P. K. Cheplogoi, "Chemical constituents in extracts from leaves of Lantana trifolia and their in vitro anti-oxidative activity," Chinese Herbal Medicines, vol. 6, no. 3, pp. 242246, 2014.

[139] A. Valerino, T. González, and I. Spengler, "Bio-directed phytochemical study of the allelopathic activity of Lantana trifolia L. leaves. Part I," in Proceedings of the XVII Congreso de la Asociación Latinoamericana de Malezas (ALAM) I Congreso Iberoamericano de Ciencia de las Malezas, IV Congreso Nacional de Ciencia de Malezas, Asociación Latinoamericana de Malezas (ALAM), Matanzas, Cuba, pp. 652-660, November 2005. 
[140] B. Abegaz, N. Asfaw, and W. Lwande, "Constituents of the essential oils from wild and cultivated Lippia adoensis Hochst. ex Walp," Journal of Essential Oil Research, vol. 5, no. 5, pp. 487-491, 1993.

[141] K. Asres and F. Bucar, "Lippia adoensis var. adoensis: studies on the essential oil composition and antioxidant activity," Ethiopian Pharmaceutical Journal, vol. 20, pp. 32-38, 2002.

[142] A. Debella, E. Makonnen, D. Abebe, F. Teka, and A. Kidanemariam, "Pain management in mice using the aqueous and ethanol extracts of four medicinal plants," East African Medical Journal, vol. 80, no. 8, pp. 435-439, 2004.

[143] A. Tesfaye, E. Makonnen, and S. Gedamu, "Hypoglycemic and antihyperglycemic activity of aqueous extract of Justicia Schimperiana leaves in normal and streptozotocin-induced diabetic mice," International Journal of Pharma Sciences and Research, vol. 7, no. 2, pp. 110-113, 2016.

[144] V. A. Da Silva, L. D. H. C. Andrade, and U. P. De Albuquerque, "Revising the cultural significance index: the case of the Fulni-ô in northeastern Brazil," Field Methods, vol. 18, no. 1, pp. 98-108, 2006.

[145] K. S. Ahmad, A. Hamid, F. Nawaz et al., "Ethnopharmacological studies of indigenous plants in Kel village, Neelum Valley, Azad Kashmir, Pakistan," Journal of Ethnobiology and Ethnomedicine, vol. 13, no. 1, p. 68, 2017.

[146] A. Bano, M. Ahmad, T. B. Hadda et al., "Quantitative ethnomedicinal study of plants used in the skardu valley at high altitude of Karakoram-Himalayan range, Pakistan," Journal of Ethnobiology and Ethnomedicine, vol. 10, no. 1, p. 43, 2014.

[147] Z. Birhanu, A. Endale, and Z. Shewamene, "An ethnomedicinal investigation of plants used by traditional healers of Gondar town, north-western Ethiopia," Journal of Medicinal Plants Studies, vol. 3, no. 2, pp. 36-43, 2015.

[148] B. Kebu, K. Ensermu, and A. Zemede, "Indigenous medicinal utilization, management and threats in Fentale area, Eastern Shewa, Ethiopia," Ethiopian Journal of Biological Sciences, vol. 3, no. 1, pp. 37-58, 2015.

[149] O. Ryding, "Lamiaceae," in Flora of Ethiopia and Eritrea, E. K. I. Hedberg, S. Edwards, S. Demissew, and E. Persson, Eds., pp. 516-604, The National Herbarium, Addis Ababa University, Addis Ababa, Ethiopia, 2006.

[150] M. Tadesse, “Asteraceae (compositae)," in Flora of Ethiopia and Eritrea, I. F. I. Hedberg and S. Edwards, Eds., pp. 222-223, Addis Ababa and Uppsala: The National Herbarium, Addis Ababa University and Uppsala University, Addis Ababa, Ethiopia, 2004. 